\title{
Two-Frequency Forced Faraday Waves: Weakly Damped Modes and Pattern Selection*
}

\author{
Mary Silber and Chad M. Topaz \\ Department of Engineering Sciences \& Applied Mathematics \\ Northwestern University \\ Evanston, IL 60208 USA \\ Anne C. Skeldon \\ Department of Mathematics \\ City University, Northampton Square \\ London, EC1V OHB, UK
}

April 17, 2000

\section{To the memory of John David Crawford}

\begin{abstract}
Recent experiments [1] on two-frequency parametrically excited surface waves produce an intriguing "superlattice" wave pattern near a codimension-two bifurcation point where both subharmonic and harmonic waves onset simultaneously, but with different spatial wavenumbers. The superlattice pattern is synchronous with the forcing, spatially periodic on a large hexagonal lattice, and exhibits small-scale triangular structure. Similar patterns have been shown to exist as primary solution branches of a generic 12 -dimensional $\mathrm{D}_{6} \dot{+} \mathrm{T}^{2}$-equivariant bifurcation problem, and may be stable if the nonlinear coefficients of the bifurcation problem satisfy certain inequalities [2]. Here we use the spatial and temporal symmetries of the problem to argue that weakly damped harmonic waves may be critical to understanding the stabilization of this pattern in the Faraday system. We illustrate this mechanism by considering the equations developed by Zhang and Viñals [3] for small amplitude, weakly damped surface waves on a semi-infinite fluid layer. We compute the relevant nonlinear coefficients in the bifurcation equations describing the onset of patterns for excitation frequency ratios of $2 / 3$ and $6 / 7$. For the $2 / 3$ case, we show that there is a fundamental difference in the pattern selection problems for subharmonic and harmonic instabilities near the codimension-two point. Also, we find that the $6 / 7$ case is significantly different from the $2 / 3$ case due to the presence of additional weakly damped harmonic modes. These additional harmonic modes can result in a stabilization of the superpatterns.
\end{abstract}

\footnotetext{
*To appear in a special issue of Physica D dedicated to the memory of John David Crawford.
} 


\section{Introduction}

Faraday waves are parametrically excited on the free surface of a fluid layer when it is subjected to a vertical vibration of sufficient strength. This pattern-forming hydrodynamic system has proven to be an especially versatile one in laboratory experiments [4, 河, exhibiting the common patterns familiar from convection (stripes, squares, hexagons, spirals), as well as more exotic patterns such as triangles [6], quasipatterns [1. 8. 8], superlattice patterns [1, 9, 10], time-dependent rhombic patterns [11] and localized waves [9, 12]. See [13] for a recent review paper on Faraday wave pattern formation.

The temporal period of the Faraday waves is typically twice that of the vibration in the case of purely sinusoidal forcing. The observation of this subharmonic response is attributed to Faraday 14 and was first explained theoretically by Benjamin and Ursell's linear stability analysis for inviscid, potential flow [15]. More recently it has been shown that waves, synchronous with the forcing, can be excited in thin layers of fluid vibrated at low frequency 16, 17, 18]; in certain viscoelastic fluids [9]; and in fluids forced periodically, but with more than one frequency component [7, 19, 20]. In each of these Faraday systems it is possible to tune the forcing parameters in order to access the transition between subharmonic and harmonic response. At this codimension-two point, both instabilities set in simultaneously, but with different spatial wavenumbers.

Many of the experimental [6, 7, 10, 11, 12, 21, 22] and theoretical studies [3, 23, 24, 25] of exotic patterns in the Faraday system attribute their formation near the codimension-two (or "bicritical") point to resonant triad interactions involving the critical or near-critical modes with different spatial wavenumbers. In particular, the focus has been on spatial triads $\mathbf{k}_{1}, \mathbf{k}_{\mathbf{2}}$ and $\mathbf{k}_{3}=\mathbf{k}_{1} \pm \mathbf{k}_{2}$, where $\left|\mathbf{k}_{1}\right|=\left|\mathbf{k}_{2}\right|$ is the wavenumber of one critical mode, and $\left|\mathbf{k}_{3}\right|$ is the wavenumber of the other critical mode. The angle $\theta_{r}$, which separates $\mathbf{k}_{1}$ and $\mathbf{k}_{2}$, is readily tuned by changing the frequency components $m \omega$ and $n \omega$ of a two-frequency periodic forcing function. It has been suggested, for example, that by tuning this angle, different types of exotic wave patterns may be selected [7]. Such a simple mechanism for nonlinear pattern selection, which is based on examining the linear instabilities of the spatially homogeneous state, is naturally attractive, but warrants careful examination as we show.

Silber and Skeldon [26] recently showed that whether or not resonant triads associated with the bicritical point affect pattern selection depends on the temporal characteristics of the competing instabilities. For instance, the bicritical point of laboratory experiments typically involves a subharmonic mode (Floquet multiplier -1 ) and a harmonic mode (Floquet multiplier +1 ). On the subharmonic side of the bicritical point, the onset pattern selection problem is strongly influenced by the presence of the weakly damped harmonic mode. In contrast, on the harmonic side, the onset pattern selection problem is completely insensitive to the presence of near critical subharmonic modes. These general ideas were demonstrated in [26] through a bifurcation analysis of a hydrodynamic model of one-dimensional Faraday waves.

Here, we extend the bifurcation analysis in [26] to two-dimensional spatially-periodic patterns and to higher forcing frequencies within the two-frequency forcing function. With the experimentally-relevant higher forcing frequencies (e.g. $6 \omega$ and $7 \omega$ ) employed in this paper, we find the new possibility that spatially-resonant triads involving nearly critical harmonic modes may influence the harmonic wave pattern selection problem. This is not an option for the lower forcing frequencies (e.g. $1 \omega / 2 \omega$ and $2 \omega / 3 \omega)$ used in previous weakly nonlinear analyses of the two-frequency Faraday problem [23, 26]. 
We follow J.D. Crawford's seminal work on Faraday waves [27, 28, 29, 30] by posing the pattern selection problem in terms of a symmetry-breaking bifurcation of the trivial fixed-point of a stroboscopic map. By restricting solutions to those that are spatially-periodic on some hexagonal lattice we obtain a finite-dimensional bifurcation problem that can be analyzed using the methods of equivariant bifurcation theory [31]. For a review of this approach to hydrodynamic pattern formation problems, see Crawford and Knobloch 32.

This formulation of the bifurcation problem allows us to address recent two-frequency Faraday wave experimental observations [1] of a transition between simple hexagons and the triangular superlattice wave pattern depicted in Figure [1]. Specifically, we follow [2] and consider a bifurcation problem that is equivariant with respect to a twelve-dimensional irreducible representation of $\mathrm{D}_{6} \dot{+} \mathrm{T}^{2}$, which is analyzed in 33 , 34. The observed harmonic wave states correspond to primary transcritical branches of the generic bifurcation problem. In order for the observed hexagonsuperlattice pattern transition to be reproduced by the bifurcation problem, we must consider a degenerate case in which the quadratic coefficient vanishes. Moreover, the cubic coefficients must satisfy certain inequalities, e.g. certain combinations of nonlinear cross-coupling coefficients must be small compared to the cubic self-coupling coefficient.

In this paper we compute the quadratic and cubic nonlinear coefficients in the bifurcation problem from the Zhang-Viñals equations [23] which apply to deep layers of low viscosity fluids subjected to a periodic acceleration. We show that the necessary inequalities for stable superlattice patterns can be satisfied for the forcing frequencies employed in the experiments $(6 \omega / 7 \omega)$, and that a resonant triad involving a weakly damped harmonic mode plays a key role in stabilizing the superpattern. Specifically, we find that the presence of a near critical harmonic mode leads to a cancellation in one of the cubic cross-coupling coefficients, causing this coefficient to become small in magnitude as required. This selects a preferred angle $\theta_{r}$ for the superlattice patterns. In other words, it suggests which of the countably infinite 12-dimensional irreducible representations of $\mathrm{D}_{6}+\mathrm{T}^{2}$ is most pertinent to this Faraday wave problem.

The paper is organized as follows. Section 2.1 presents background linear stability results for the two-frequency Faraday experiment, while section 2.2 reviews results from [26] on the influence of spatio-temporally resonant triads on pattern selection. Section 2.3 then formulates the generic bifurcation problem relevant to our investigation. The bifurcation results derived from the twofrequency Faraday problem modeled by the Zhang-Viñals equations are presented in Section 3; the coefficients of the leading nonlinear terms are evaluated numerically from expressions derived perturbatively in the Appendix. We consider two different cases. In Section 3.2 we consider an example involving forcing frequencies in ratio $m / n=2 / 3$, focusing on differences between the pattern selection problems for subharmonic and harmonic wave onset in a vicinity of the bicritical point. Section 3.3 then turns to an example involving higher forcing frequencies in ratio $m / n=6 / 7$, and shows how weakly damped harmonic modes can stabilize harmonic wave superpatterns involving

the angle $\theta_{r}$ associated with a harmonic wave resonant triad. Finally, Section 1 concludes the paper with a brief summary of our results and some discussion of issues we hope to address in the future.

\section{Background}




\section{$2.1 \quad$ Linear Results}

In the two-frequency Faraday wave problem a container of fluid is accelerated in the vertical direction with an excitation of the form

$$
g(t)=g_{0}+g_{z}(\cos (\chi) \cos (m \omega t)+\sin (\chi) \cos (n \omega t+\phi)) .
$$

Here $m$ and $n$ are co-prime integers, so the forcing function is periodic with period $T=\frac{2 \pi}{\omega}$, and $g_{0}$ is the usual gravitational acceleration. For small amplitude acceleration $g_{z}$ the surface of the fluid remains flat and the fluid layer is merely translated up and down with the drive. For higher values of $g_{z}$ waves are parametrically excited on the surface of the fluid layer.

Besson, Edwards and Tuckerman [20], starting with the Navier-Stokes equations for the free boundary problem, determined the linear stability of the flat surface in the case that the fluid layer has finite depth but is unbounded horizontally. They used a Floquet-Fourier ansatz and solved

the linear stability problem numerically to determine, for each spatial wavenumber $k$, the value of $g_{z}$ where a Floquet multiplier first crosses the unit circle. The resulting neutral stability curves show that the primary instability is to either subharmonic or harmonic waves depending on the value of $\chi$ and the values of $m$ and $n$. (Harmonic/subharmonic response is relative to the forcing period $T=2 \pi / \omega$.) Typically, if $\chi$ is small so that $\cos (\chi) \cos (m \omega t)$ is of greater significance than $\sin (\chi) \cos (n \omega t+\phi)$, then the response is harmonic if $m$ is even and subharmonic if $m$ is odd. Similarly, if $\chi$ is close to $\pi / 2$, the primary instability is (sub)harmonic if $n$ is even (odd). At the socalled bicritical point, $\chi=\chi_{c}$, both harmonic and subharmonic instabilities onset at the same value of the excitation amplitude, but with different wavenumbers. The harmonic superlattice pattern of Figure [1a, observed by Kudrolli, Pier and Gollub [1], was obtained near the bicritical point for $m / n=6 / 7$ forcing in (11). The pertinent neutral stability curve, computed using the experimental fluid parameters, is given in Figure il $\mathrm{b}$.

\subsection{Spatio-Temporally Resonant Triads}

When the hydrodynamic problem is posed on a horizontally unbounded domain there is no preferred direction (in the horizontal) so that each critical wavenumber from linear analysis actually corresponds to a circle of critical wavevectors. There are two such critical circles at the bicritical point, as shown in Figure 2. In this situation it has been argued that resonant triads may play a central role in the Faraday wave pattern selection problem [7, 6, 22, 23, 24. Resonant triads are comprised of three critical wavevectors that sum to zero; two examples are shown in Figure 2. In the first example, $\mathbf{k}_{m_{1}}+\mathbf{k}_{m_{2}}-\mathbf{k}_{n}=\mathbf{0}$, and in the second example $\mathbf{k}_{n_{1}}-\mathbf{k}_{n_{2}}-\mathbf{k}_{m}=\mathbf{0}$. Here the $m, n$ subscripts indicate that the critical wavenumbers can be roughly associated with the $m \omega$ and $n \omega$ excitation terms in (1). We identify with each resonant triad an angle $\theta_{r} \in\left(0, \frac{\pi}{2}\right]$, which separates the critical wavevectors with the same length. For instance, the angle in Figure 2ab satisfies

$$
\cos \left(\frac{\theta_{r}}{2}\right)=\frac{k_{n}}{2 k_{m}}
$$

while the angle in Figure 20 satisfies

$$
\sin \left(\frac{\theta_{r}}{2}\right)=\frac{k_{m}}{2 k_{n}}
$$




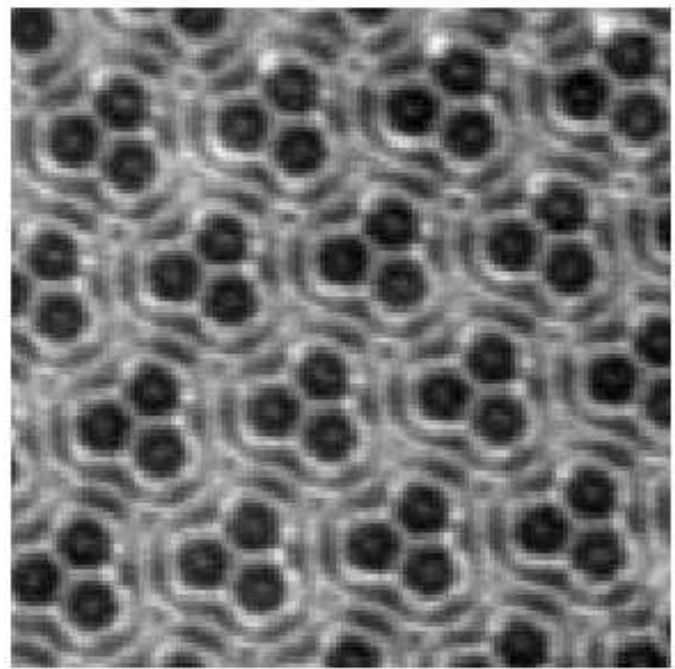

(a)

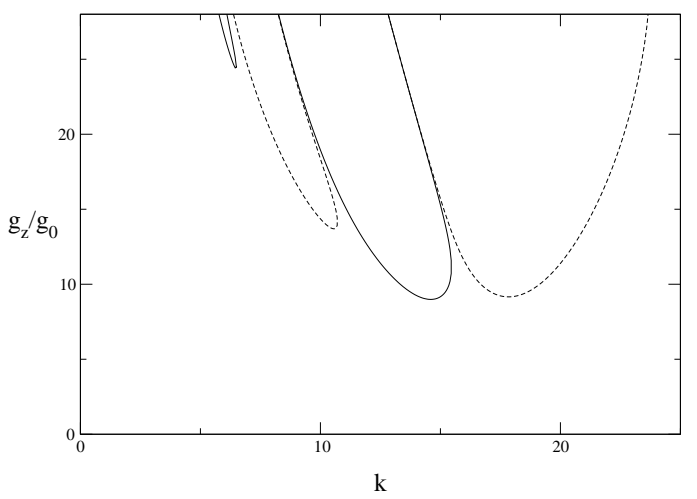

(b)

Figure 1: (a) Blow up of the experimental superlattice Faraday wave pattern described in [1] (courtesy of Kudrolli, Pier and Gollub). The forcing function (1) has $m / n=6 / 7, \chi=61^{\circ}$ and $\phi=20^{\circ}$. Note that the pattern is periodic on a (large) hexagonal lattice, and that in each hexagonal 'tile' there is small triangular structure. (b) The corresponding neutral stability curve, calculated from the full (linearized) hydrodynamic equations, for the experimental parameters reported in [1]. (Sub)harmonic resonance tongues are given by solid (dashed) lines. The neutral curves are computed using the method described in [20].

(a)

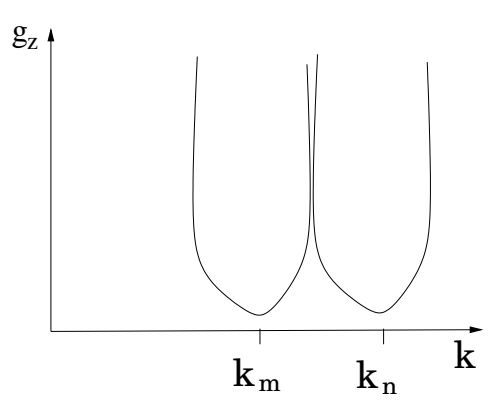

(b)

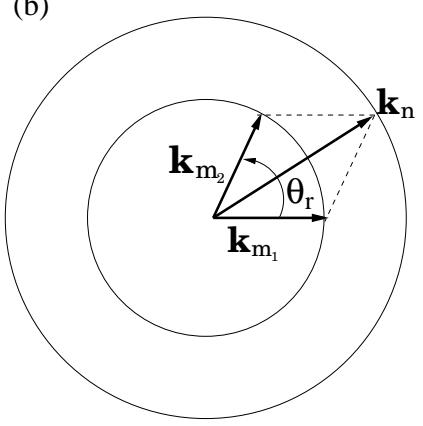

(c)

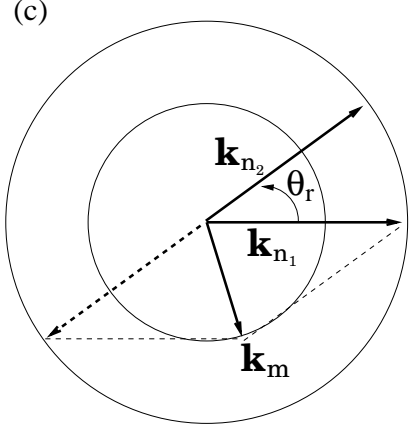

Figure 2: (a) A plot of a neutral stability curve $g_{z}(k)$ showing minima at $k=k_{m}$ and $k=k_{n}$. (b) An associated spatially resonant triad $\mathbf{k}_{m_{1}}, \mathbf{k}_{m_{2}}$ and $\mathbf{k}_{n}=\mathbf{k}_{m_{1}}+\mathbf{k}_{m_{2}}$. (c) An associated spatially resonant triad $\mathbf{k}_{n_{1}}, \mathbf{k}_{n_{2}}$ and $\mathbf{k}_{m}=\mathbf{k}_{n_{1}}-\mathbf{k}_{n_{2}}$. 
To illustrate the potential for resonant triads to influence pattern formation in parametrically excited systems we consider a bifurcation problem involving the three critical Fourier modes associated with the resonant triads of Figure 2. Much of this discussion is a review of the key theoretical ideas in 26]. Because of the periodic forcing of the system, it is natural to formulate the bifurcation problem in terms of a stroboscopic map [27]. Specifically, we denote the free surface height $z=h(\mathbf{x}, t)\left(\mathbf{x} \in \mathbf{R}^{2}\right)$ at time $t=p T(p \in \mathbf{Z})$ by

$$
h(\mathbf{x}, p T)=A(p) e^{i \mathbf{k}_{l_{1}} \cdot \mathbf{x}}+B(p) e^{i \mathbf{k}_{l_{2}} \cdot \mathbf{x}}+C(p) e^{i\left(\mathbf{k}_{l_{1}}+\mathbf{k}_{l_{2}}\right) \cdot \mathbf{x}}+\text { c.c. }+\cdots .
$$

Here $A, B$ and $C$ are the complex amplitudes of the linear modes that are neutrally stable at the bicritical point and which form a resonant triad. In this discussion we assume that the angle $\theta_{r}$ between $\mathbf{k}_{l_{1}}$ and $\mathbf{k}_{l_{2}}$ is not $\pi / 3$ so that the critical modes interact nonlinearly to generate other modes on a rhombic (rather than hexagonal) lattice. These additional modes, denoted by $\cdots$ above, are linearly damped at the bicritical point. We may then use the spatial reflection and translation symmetries to determine the general form of the bifurcation equations that govern the dynamics on a center manifold. Specifically, to cubic order, the codimension-two bifurcation problem takes the form

$$
\begin{aligned}
& A \rightarrow \sigma A+\alpha \bar{B} C+\left(a|A|^{2}+b|B|^{2}+c|C|^{2}\right) A \\
& B \rightarrow \sigma B+\alpha \bar{A} C+\left(a|B|^{2}+b|A|^{2}+c|C|^{2}\right) B \\
& C \rightarrow \mu C+\delta A B+\left(d|A|^{2}+d|B|^{2}+e|C|^{2}\right) C,
\end{aligned}
$$

where $\bar{A}$ is the complex conjugate of $A$, and the coefficients are all real. The Floquet multipliers $\sigma$ and $\mu$ are either +1 or -1 depending on whether the linear modes $A, B$, and $C$ are harmonically or subharmonically excited, respectively.

In deriving (5) we considered only the spatial symmetries associated with the resonant triad. Following [27, we enforce the temporal symmetry associated with the triad through a normal form transformation of (5). Specifically, there exists a near-identity nonlinear transformation that removes all nonlinear terms in (5) which do not commute with $L^{T}$, where $L$ is the Jacobian matrix associated with the linearized problem (see, for example, Crawford's review paper on bifurcation theory 35). Here

$$
L=\left(\begin{array}{ccc}
\sigma & 0 & 0 \\
0 & \sigma & 0 \\
0 & 0 & \mu
\end{array}\right),
$$

where $|\sigma|=|\mu|=1$. The normal form symmetry may be interpreted in terms of time-translation. Specifically, advancing by one period in time maps period-doubled modes to their negatives, e.g. if $\mu=-1$, then advancing one period takes $C \rightarrow-C$.

In the case that $\mu=+1(\sigma= \pm 1)$, the bifurcation problem (5) is already in normal form. This observation is trivial if $\sigma=+1$. If $\sigma=-1$, then the normal form symmetry is equivalent in action to that associated with the spatial translation symmetry $\mathbf{x} \rightarrow \mathbf{x}+\mathbf{d}$, where $\mathbf{d}$ satisfies $\mathbf{k}_{l_{1}} \cdot \mathbf{d}=\mathbf{k}_{l_{2}} \cdot \mathbf{d}=\pi$.

In contrast, in the case that $\mu=-1$, a normal form transformation removes the quadratic terms in the bifurcation problem (5). The normal form of the bifurcation problem, through cubic order, is then

$$
A \quad \rightarrow \quad \sigma A+\left(a|A|^{2}+b|B|^{2}+c|C|^{2}\right) A
$$




$$
\begin{aligned}
& B \rightarrow \sigma B+\left(a|B|^{2}+b|A|^{2}+c|C|^{2}\right) B \\
& C \rightarrow-C+\left(d|A|^{2}+d|B|^{2}+e|C|^{2}\right) C .
\end{aligned}
$$

We note that $C=0$ is a dynamically-invariant subspace of (7). This is true to all orders of the normal form since $C=0$ is the fixed point subspace of a (spatio-)temporal symmetry. Specifically, if $\sigma=+1$ then $C=0$ is the fixed point subspace associated with the time translation by one-period, i.e., $(A, B, C) \rightarrow(A, B,-C)$. And if $\sigma=-1$, then $C=0$ is the fixed-point subspace associated with the spatio-temporal symmetry involving time translation by one period followed by spatial translation by $\mathbf{d}$, where again $\mathbf{k}_{l_{1}} \cdot \mathbf{d}=\mathbf{k}_{l_{2}} \cdot \mathbf{d}=\pi$.

We now examine (5) more closely in the case that $\mu=+1$ so that we cannot remove the quadratic nonlinearities by normal form transformation. We focus on a detuning from the bicritical point such that the $C$ mode is weakly damped, while the $A, B$ modes are neutrally stable. In this case, $|\sigma|=1, \mu<1$, we can further reduce the bifurcation problem to one involving the critical modes $A$ and $B$, with $C$ constrained to the center manifold: $C=\frac{\delta}{(1-\mu)} A B+\cdots$. We then obtain the reduced bifurcation problem

$$
\begin{aligned}
& A \rightarrow \sigma A+a|A|^{2} A+\beta\left(\theta_{r}\right)|B|^{2} A \\
& B \rightarrow \sigma B+a|B|^{2} B+\beta\left(\theta_{r}\right)|A|^{2} B,
\end{aligned}
$$

where the cross-coupling coefficient is

$$
\beta\left(\theta_{r}\right)=b+\frac{\alpha \delta}{(1-\mu)} .
$$

We see that in this case, the near critical spatio-temporally resonant mode $C$ in (5) can contribute significantly to the cross-coupling coefficient $\beta\left(\theta_{r}\right)$ since $0<1-\mu \ll 1$ in (9). For example, for $\mu$ sufficiently close to 1 , the second term in (9) dominates and $\beta\left(\theta_{r}\right)$ becomes large in magnitude. However, we also point out that if $b$ and $\alpha \delta$ have opposite signs, then $\beta\left(\theta_{r}\right)$ could actually vanish for some $\mu-1>0$. Examples of these two very different situations are given in sections 3.2 and 3.3 , respectively.

We contrast the above with what happens when $\mu=-1$ at the bicritical point. In this case $\alpha=\delta=0$ in the normal form (7) and $C=0$ is an invariant subspace with associated dynamics of the form (8) with $\beta\left(\theta_{r}\right)=b$. In this case, the triad is spatially resonant, but not temporally resonant, and the cross-coupling coefficient is insensitive to any parameter proximity to the bicritical point.

These observations about $\beta\left(\theta_{r}\right)$ are important for understanding which patterns might be observable near onset since branching direction and stability of patterns are determined by various nonlinear (cross-coupling) coefficients in the amplitude equations. We discuss this further at the end of Section 2.3.

Finally we note that similar results to the $\mu=-1$ case above apply when there are weakly damped modes with complex Floquet multipliers. Specifically, these modes do not contribute significantly to the cubic cross-coupling coefficient $\beta(\theta)$, even when they are spatially resonant with the critical modes. Only damped modes with Floquet multiplier $\mu$ sufficiently close to +1 contribute. 


\subsection{Hexagonal Lattice Bifurcation Problem}

The analysis of the previous section led to certain conclusions about the nonlinear coefficients in the general rhombic lattice bifurcation problem

$$
\begin{aligned}
& v_{1} \rightarrow \sigma v_{1}+\left(a\left|v_{1}\right|^{2}+\beta(\theta)\left|v_{2}\right|^{2}\right) v_{1} \\
& v_{2} \rightarrow \sigma v_{2}+\left(a\left|v_{2}\right|^{2}+\beta(\theta)\left|v_{1}\right|^{2}\right) v_{2} .
\end{aligned}
$$

Here $v_{1}, v_{2}$ are the complex amplitudes of two critical Fourier modes with wavevectors $\mathbf{k}_{1}, \mathbf{k}_{2}$ $\left(\left|\mathbf{k}_{1}\right|=\left|\mathbf{k}_{2}\right|=k_{c}\right)$ that are separated by an angle $\theta \in\left(0, \frac{\pi}{2}\right]\left(\theta \neq \frac{\pi}{3}\right)$. In particular, it follows from (9) that if a weakly damped harmonic mode is removed via center manifold reduction, then $\beta(\theta)$ may become large in magnitude when the spatial resonance condition is met, i.e. when $\theta=\theta_{r}$. This is in contrast to the situation where there are weakly damped subharmonic modes, which have no special influence on the pattern selection problem at onset.

We now lay the framework for examining possible implications of these results for stability of harmonic hexagonal and triangular superpatterns. We follow [2] and introduce the twelve-dimensional $\mathrm{D}_{6}+\mathrm{T}^{2}$-equivariant bifurcation problems that enable us to determine the relative stability of simple

hexagonal patterns, stripe patterns and certain rhombic and superlattice patterns. We make use of bifurcation results derived in [2, 33, 34], which apply when there is a single critical wavenumber $k_{c}$, to demonstrate how the magnitude of the cross-coupling terms are pivotal in determining pattern stability. As before, we consider a stroboscopic map, but now restrict analysis to patterns that are doubly-periodic on some hexagonal lattice. For instance, the free surface height takes the form

$$
h(\mathbf{x}, p T)=\sum_{\mathbf{m} \in \mathbf{Z}^{2}} \hat{h}_{\mathbf{m}}(p) e^{i\left(m_{1} \mathbf{k}_{1}+m_{2} \mathbf{k}_{2}\right) \cdot \mathbf{x}}+\text { c.c. }
$$

at time $t=p T$, where $\mathbf{k}_{1}, \mathbf{k}_{2} \in \mathbf{R}^{2}$ generate a hexagonal dual lattice $\left(\left|\mathbf{k}_{1}\right|=\left|\mathbf{k}_{2}\right|\right.$ and $\mathbf{k}_{1} \cdot \mathbf{k}_{2}=$ $\left.-\frac{1}{2}\left|\mathbf{k}_{1}\right|^{2}\right)$; see Figure 3 .

The twelve-dimensional irreducible representations of $\mathrm{D}_{6} \dot{+} \mathrm{T}^{2}$ apply to the bifurcation problem when there are twelve integer pairs $\left(m_{1}, m_{2}\right)$ in (11) such that $\left|m_{1} \mathbf{k}_{1}+m_{2} \mathbf{k}_{2}\right|=k_{c}$, where $k_{c}$ is the critical wavenumber of the instability at the bifurcation point. See Figure 3 for an example. Following [33] we will associate with each twelve-dimensional irreducible representation an integer pair $\left(n_{1}, n_{2}\right)$; in particular $n_{1}$ and $n_{2}$ are co-prime, $n_{1}>n_{2}>n_{1} / 2>0$, and $n_{1}+n_{2}$ is not a multiple of 3 . The neutral modes that span the center eigenspace at the bifurcation point take the form

$$
\left\{z_{1} e^{i \mathbf{K}_{1} \cdot \mathbf{x}}+z_{2} e^{i \mathbf{K}_{2} \cdot \mathbf{x}}+z_{3} e^{i \mathbf{K}_{3} \cdot \mathbf{x}}+z_{4} e^{i \mathbf{K}_{4} \cdot \mathbf{x}}+z_{5} e^{i \mathbf{K}_{5} \cdot \mathbf{x}}+z_{6} e^{i \mathbf{K}_{6} \cdot \mathbf{x}}+\text { c.c. } \mid z_{j} \in \mathbf{C}\right\},
$$

where

$$
\begin{aligned}
\mathbf{K}_{1}=n_{1} \mathbf{k}_{1}+n_{2} \mathbf{k}_{2}, & \mathbf{K}_{4}=n_{1} \mathbf{k}_{1}+\left(n_{1}-n_{2}\right) \mathbf{k}_{2}, \\
\mathbf{K}_{2}=\left(-n_{1}+n_{2}\right) \mathbf{k}_{1}-n_{1} \mathbf{k}_{2}, & \mathbf{K}_{5}=-n_{2} \mathbf{k}_{1}-n_{1} \mathbf{k}_{2}, \\
\mathbf{K}_{3}=-n_{2} \mathbf{k}_{\mathbf{1}}+\left(n_{1}-n_{2}\right) \mathbf{k}_{2}, & \mathbf{K}_{6}=\left(n_{2}-n_{1}\right) \mathbf{k}_{\mathbf{1}}+n_{2} \mathbf{k}_{2} .
\end{aligned}
$$

Note that $\pm \mathbf{K}_{1}, \pm \mathbf{K}_{2}, \pm \mathbf{K}_{3}$ point to the vertices of a hexagon, as do $\pm \mathbf{K}_{4}, \pm \mathbf{K}_{5}, \pm \mathbf{K}_{6}$, and that the two hexagons are rotated relative to each other by an angle $\theta_{h} \in\left(0, \frac{\pi}{3}\right)$ indicated in Figure 3. This angle is related to $\left(n_{1}, n_{2}\right)$ by

$$
\cos \left(\theta_{h}\right)=\frac{n_{1}^{2}+2 n_{1} n_{2}-2 n_{2}^{2}}{2\left(n_{1}^{2}-n_{1} n_{2}+n_{2}^{2}\right)}
$$




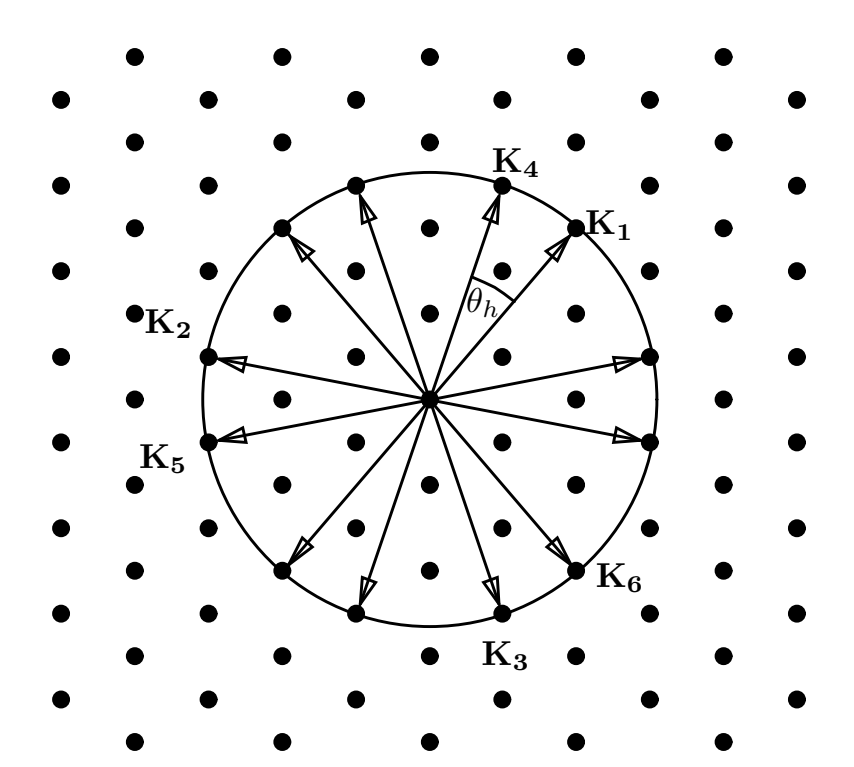

Figure 3: Hexagonal $\mathbf{k}$-space lattice, with critical circle of radius $k_{c}$ superimposed. In this example $\left(n_{1}, n_{2}\right)=(3,2)$ in $(13)$, and the critical circle intersects twelve points that lie at the vertices of two hexagons rotated by $\theta_{h}$ relative to each other.

Also note that the ratio of lengthscales for superpatterns depends on $\left(n_{1}, n_{2}\right)$. Specifically, $\left|\mathbf{k}_{1}\right|$ determines the larger periodicity scale of the superpatterns, while $\left|\mathbf{K}_{j}\right|=k_{c}$ determines the smaller lengthscale associated with the instability; thus the lengthscale ratio is

$$
\left|\mathbf{K}_{j}\right| /\left|\mathbf{k}_{1}\right|=\sqrt{n_{1}^{2}-n_{1} n_{2}+n_{2}^{2}} \geq \sqrt{7} .
$$

The example of Figure 3 corresponds to $\left(n_{1}, n_{2}\right)=(3,2)$, for which $\theta_{h} \approx 22^{\circ}$ in (14) and the lengthscale ratio 15 is is the smallest associated with a hexagonal lattice, namely $\sqrt{7}$. These are the angle and lengthscale ratio that apply to the experimental superlattice pattern reproduced from [1] in Figure 1 a.

The general form of the twelve-dimensional $\mathrm{D}_{6}+\mathrm{T}^{2}$-equivariant mappings are derived in [34]. Through cubic order in $z_{j}$, they take the form

$$
\begin{aligned}
z_{1} & \rightarrow \sigma\left((1+\lambda) z_{1}+\epsilon \bar{z}_{2} \bar{z}_{3}+\left(b_{1}\left|z_{1}\right|^{2}+b_{2}\left|z_{2}\right|^{2}+b_{2}\left|z_{3}\right|^{2}+b_{4}\left|z_{4}\right|^{2}+b_{5}\left|z_{5}\right|^{2}+b_{6}\left|z_{6}\right|^{2}\right) z_{1}\right) \\
z_{2} & \rightarrow \sigma\left((1+\lambda) z_{2}+\epsilon \bar{z}_{1} \bar{z}_{3}+\left(b_{1}\left|z_{2}\right|^{2}+b_{2}\left|z_{1}\right|^{2}+b_{2}\left|z_{3}\right|^{2}+b_{4}\left|z_{5}\right|^{2}+b_{5}\left|z_{6}\right|^{2}+b_{6}\left|z_{4}\right|^{2}\right) z_{2}\right) \\
z_{3} & \rightarrow \sigma\left((1+\lambda) z_{3}+\epsilon \bar{z}_{1} \bar{z}_{2}+\left(b_{1}\left|z_{3}\right|^{2}+b_{2}\left|z_{1}\right|^{2}+b_{2}\left|z_{2}\right|^{2}+b_{4}\left|z_{6}\right|^{2}+b_{5}\left|z_{4}\right|^{2}+b_{6}\left|z_{5}\right|^{2}\right) z_{3}\right) \\
z_{4} & \rightarrow \sigma\left((1+\lambda) z_{4}+\epsilon \bar{z}_{5} \bar{z}_{6}+\left(b_{1}\left|z_{4}\right|^{2}+b_{2}\left|z_{5}\right|^{2}+b_{2}\left|z_{6}\right|^{2}+b_{4}\left|z_{1}\right|^{2}+b_{5}\left|z_{3}\right|^{2}+b_{6}\left|z_{2}\right|^{2}\right) z_{4}\right) \\
z_{5} & \rightarrow \sigma\left((1+\lambda) z_{5}+\epsilon \bar{z}_{4} \bar{z}_{6}+\left(b_{1}\left|z_{5}\right|^{2}+b_{2}\left|z_{4}\right|^{2}+b_{2}\left|z_{6}\right|^{2}+b_{4}\left|z_{2}\right|^{2}+b_{5}\left|z_{1}\right|^{2}+b_{6}\left|z_{3}\right|^{2}\right) z_{5}\right) \\
z_{6} & \rightarrow \sigma\left((1+\lambda) z_{6}+\epsilon \bar{z}_{4} \bar{z}_{5}+\left(b_{1}\left|z_{6}\right|^{2}+b_{2}\left|z_{4}\right|^{2}+b_{2}\left|z_{5}\right|^{2}+b_{4}\left|z_{3}\right|^{2}+b_{5}\left|z_{2}\right|^{2}+b_{6}\left|z_{1}\right|^{2}\right) z_{6}\right),
\end{aligned}
$$

where $\lambda$ measures the distance from the critical excitation amplitude, and $\sigma=+1(-1)$ in the case of (sub)harmonic instability. All nonlinear coefficients are real. If $\sigma=-1$ then a normal 
form transformation removes all even terms on the right-hand-side of (16) and hence $\epsilon=0$. The dependence of the general equivariant bifurcation problem on $\left(n_{1}, n_{2}\right)$ does not appear until higher than cubic order in its Taylor expansion [34].

We now recall some basic results pertaining to the bifurcation problem (16). In the $\sigma=+1$ case the equivariant branching lemma [31] ensures the existence of harmonic wave solution branches in the form of stripes, simple hexagons, rhombs, and super hexagons [33]. A primary solution branch with submaximal isotropy, named super triangles, was also shown to exist in [2]. See Figure 11a for an example of this pattern. Table 1 gives the general form of these solutions, along with their branching and stability assignments. The general bifurcation results in the case that $\sigma=-1$ can be found in [34]; this bifurcation problem differs from the harmonic case in that it possesses an additional $\mathrm{Z}_{2}$ normal form symmetry. The equivariant branching lemma then ensures existence of five additional solution branches to those listed in Table 1 [ [34.

The generic presence of a quadratic term in (16) for the harmonic case renders all of the solutions in Table 1 unstable at bifurcation. Hence the transition from the flat state to the patterned harmonic wave state is expected to be hysteretic. In order to capture stable weakly nonlinear solutions, we must focus our analysis on the unfolding of the degenerate bifurcation problem $\epsilon=0$. Note that when $\epsilon=0$ the stability of simple and super hexagons/triangles is not determined at cubic order since the phases $\phi_{j}$ of solutions $z_{j}=r_{j} e^{i \phi_{j}}$ to (16) are then arbitrary. Even in the case of $0<|\epsilon| \ll 1$ the relative stability of super hexagons and super triangles depends on terms that are at least fifth order. However, we may use the cubic truncation to determine that one (and only one) of these two solutions is stable. The higher order terms are only needed to determine whether it is the hexagonal or triangular superpattern [2].

When $0<|\epsilon| \ll 1$, it follows from Table 1 that a necessary condition for one of the superpatterns to be stable over some range of $\lambda$ values near onset is for

$$
b_{1}+2 b_{2}<-\left|b_{4}+b_{5}+b_{6}\right|<0 .
$$

The combination $b_{1}+2 b_{2}$ is independent of the lattice angle $\theta_{h}$ in (14); it is computed from a hydrodynamic model of the two-frequency Faraday problem in the Appendix by considering bifurcation to simple hexagons. In contrast, the combination $b_{4}+b_{5}+b_{6}$ depends on $\theta_{h}$ and is computed in the appendix from the hydrodynamic equations by considering the rhombic lattice bifurcation problem (10). Specifically, the cross-coupling coefficients $b_{4}, b_{5}, b_{6}$ are

$$
b_{4}=\beta\left(\theta_{h}\right), \quad b_{5}=\beta\left(\theta_{h}+\frac{2 \pi}{3}\right), \quad b_{6}=\beta\left(\theta_{h}-\frac{2 \pi}{3}\right),
$$

where $\theta_{h}$ is the angle between $\mathbf{K}_{1}$ and $\mathbf{K}_{\mathbf{4}}$ given by (14). (The function $\beta(\theta)$ may be extended from $\theta \in\left(0, \frac{\pi}{2}\right]$ to angles $\theta \in(0,2 \pi)$ using $\beta(\theta)=\beta(-\theta)=\beta(\theta+\pi)$, identities that follow from the symmetries of the rhombic lattice bifurcation problem.)

The inequality (17) will be satisfied (if at all) only for those $\theta_{h}$ values where $\left|b_{4}+b_{5}+b_{6}\right|$ is small compared to $\left|b_{1}+2 b_{2}\right|$. Moreover, if $b_{1}-b_{2}<0$ in addition to (17), then simple hexagons become unstable on a given hexagonal lattice when

$$
\lambda=-\frac{\epsilon^{2}\left(b_{4}+b_{5}+b_{6}\right)}{\left(b_{1}+2 b_{2}-b_{4}-b_{5}-b_{6}\right)^{2}} .
$$

If $b_{4}+b_{5}+b_{6}<0$ for all $\theta_{h}$, then simple hexagons first lose stability with increasing $\lambda$ to a perturbation in the direction of a superpattern for that value of $\theta_{h}$ that minimizes $\left|b_{4}+b_{5}+b_{6}\right|$. 
Table 1: Branching equations and stability assignments for the harmonic case $(\sigma=+1) ; \epsilon, b_{1}, \ldots, b_{6}$ are coefficients in the bifurcation equations (16). A solution is stable if all quantities in the right column are negative. See [2, 33, 34] for more details.

\begin{tabular}{|c|c|}
\hline Planform and branching equation & Stability \\
\hline $\begin{array}{c}\text { Stripes } \\
\mathbf{z}=(x, 0,0,0,0,0) \\
0=\lambda x+b_{1} x^{3}+\mathcal{O}\left(x^{5}\right)\end{array}$ & $\begin{array}{c}\operatorname{sgn}\left(b_{1}\right), \\
\operatorname{sgn}\left(\epsilon x+\left(b_{2}-b_{1}\right) x^{2}\right), \quad \operatorname{sgn}\left(-\epsilon x+\left(b_{2}-b_{1}\right) x^{2}\right), \\
\operatorname{sgn}\left(b_{4}-b_{1}\right), \quad \operatorname{sgn}\left(b_{5}-b_{1}\right), \quad \operatorname{sgn}\left(b_{6}-b_{1}\right)\end{array}$ \\
\hline $\begin{array}{c}\text { Simple Hexagons } \\
\mathbf{z}=(x, x, x, 0,0,0) \\
0=\lambda x+\epsilon x^{2}+\left(b_{1}+2 b_{2}\right) x^{3}+\mathcal{O}\left(x^{4}\right)\end{array}$ & $\begin{array}{c}\operatorname{sgn}\left(\epsilon x+2\left(b_{1}+2 b_{2}\right) x^{2}\right), \quad \operatorname{sgn}\left(-\epsilon x+\left(b_{1}-b_{2}\right) x^{2}\right) \\
\operatorname{sgn}\left(-\epsilon x+\left(b_{4}+b_{5}+b_{6}-b_{1}-2 b_{2}\right) x^{2}\right) \\
\operatorname{sgn}\left(-\epsilon x+\mathcal{O}\left(x^{3}\right)\right)\end{array}$ \\
\hline $\begin{array}{c}\text { Rhombs }\left(\mathrm{Rh}_{4}\right) \\
\mathbf{z}=(x, 0,0, x, 0,0) \\
0=\lambda x+\left(b_{1}+b_{4}\right) x^{3}+\mathcal{O}\left(x^{5}\right)\end{array}$ & $\begin{array}{c}\operatorname{sgn}\left(b_{1}+b_{4}\right), \quad \operatorname{sgn}\left(b_{1}-b_{4}\right), \quad \operatorname{sgn}\left(\zeta_{1}\right), \quad \operatorname{sgn}\left(\zeta_{2}\right), \\
\text { where } \zeta_{1}+\zeta_{2}=\left(-2 b_{1}-2 b_{4}+2 b_{2}+b_{5}+b_{6}\right) x^{2}, \\
\zeta_{1} \zeta_{2}=-\epsilon^{2} x^{2}+\left(b_{1}+b_{4}-b_{2}-b_{5}\right)\left(b_{1}+b_{4}-b_{2}-b_{6}\right) x^{4}\end{array}$ \\
\hline $\begin{array}{c}\text { Rhombs }\left(\mathrm{Rh}_{5}\right) \\
\mathbf{z}=(x, 0,0,0, x, 0)\end{array}$ & same as $\mathrm{Rh}_{4}$ with $b_{4} \leftrightarrow b_{5}$ \\
\hline $\begin{array}{c}\text { Rhombs }\left(\mathrm{Rh}_{6}\right) \\
\mathbf{z}=(x, 0,0,0,0, x)\end{array}$ & same as $\mathrm{Rh}_{4}$ with $b_{4} \leftrightarrow b_{6}$ \\
\hline $\begin{array}{c}\text { Super Hexagons } \\
\mathbf{z}=(x, x, x, x, x, x) \\
0=\lambda x+\epsilon x^{2}+\left(b_{1}+2 b_{2}\right) x^{3} \\
+\left(b_{4}+b_{5}+b_{6}\right) x^{3}+\mathcal{O}\left(x^{4}\right)\end{array}$ & $\begin{array}{c}\operatorname{sgn}\left(\epsilon x+2\left(b_{1}+2 b_{2}+b_{4}+b_{5}+b_{6}\right) x^{2}\right) \\
\operatorname{sgn}\left(\epsilon x+2\left(b_{1}+2 b_{2}-b_{4}-b_{5}-b_{6}\right) x^{2}\right) \\
\operatorname{sgn}\left(-\epsilon x+\mathcal{O}\left(x^{3}\right)\right), \quad \operatorname{sgn}\left(\zeta_{1}\right), \quad \operatorname{sgn}\left(\zeta_{2}\right), \\
\text { where } \zeta_{1}+\zeta_{2}=-4 \epsilon x+4\left(b_{1}-b_{2}\right) x^{2}, \\
\zeta_{1} \zeta_{2}=4\left(\epsilon x-\left(b_{1}-b_{2}\right) x^{2}\right)^{2} \\
\left.\quad-2\left(\left(b_{4}-b_{5}\right)^{2}+\left(b_{4}-b_{6}\right)^{2}+\left(b_{5}-b_{6}\right)^{2}\right)\right) x^{4} \\
\operatorname{sgn}\left(\zeta_{3}\right), \text { where } \zeta_{3}=\mathcal{O}\left(x^{2\left(n_{1}-1\right)}\right)\end{array}$ \\
\hline $\begin{array}{l}\text { Super Triangles } \\
\mathbf{z}=(z, z, z, z, z, z) \\
z=x e^{i \psi}, \psi \neq 0, \pi, \ldots\end{array}$ & $\begin{array}{l}\text { Same as super hexagons } \\
\text { except } \zeta_{3} \rightarrow-\zeta_{3}\end{array}$ \\
\hline
\end{tabular}



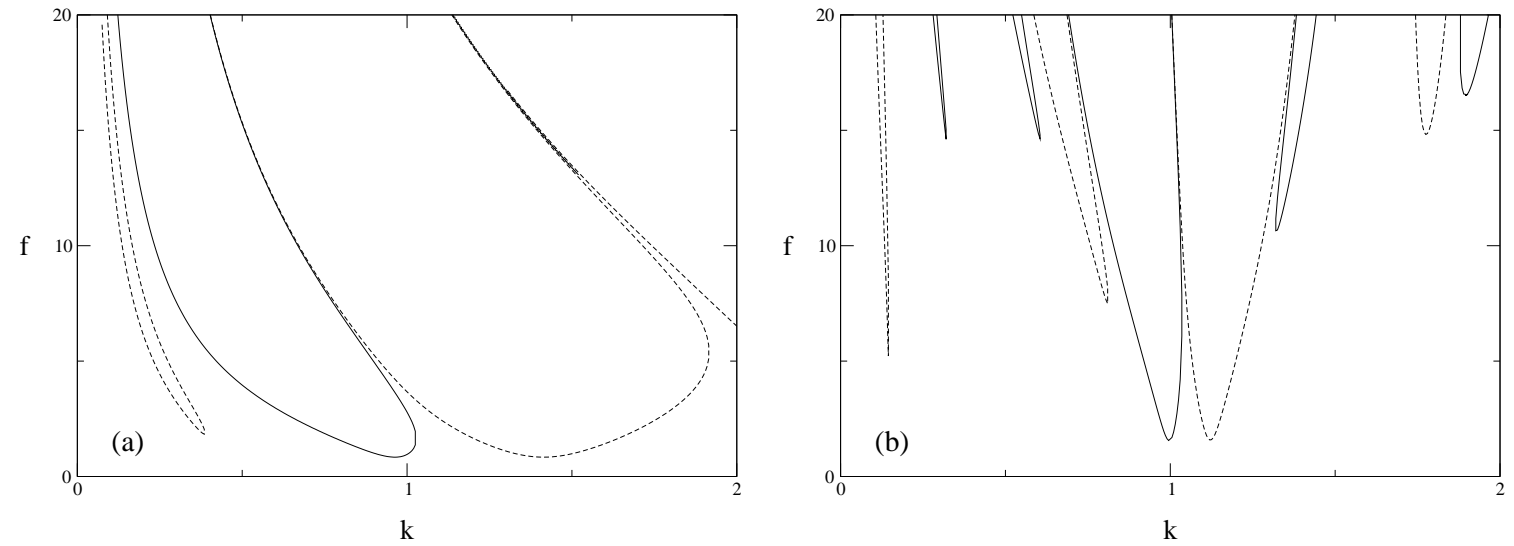

Figure 4: Neutral stability curves computed from (20) linearized about $h=\Phi=0$. Floquet multipliers of $+1(-1)$ are indicated by solid (dashed) lines. (a) $m / n=2 / 3, \phi=0^{\circ}, \chi=\chi_{c}=66.6^{\circ}$, $\Gamma_{0}=0.53, G_{0}=0.47$ and $\gamma=0.09$ in (20)-(21). (b) $m / n=6 / 7, \phi=0^{\circ}, \chi=\chi_{c}=53.0^{\circ}, \Gamma_{0}=7.5$, $G_{0}=1.5$ and $\gamma=0.08$.

If $b_{4}+b_{5}+b_{6}>0$ for any $\theta_{h}$, then small amplitude simple hexagons are unstable when $\lambda>0$. Thus we expect the stability properties of superpatterns and simple hexagons to be affected by the presence of a weakly damped harmonic mode when $\theta_{h}$ or $\theta_{h} \pm 2 \pi / 3$ is near $\theta_{r}$ (or $\pi \pm \theta_{r}$ ), the resonant triad angle, since it is in this situation that one of the cross-coupling coefficients $b_{4}, b_{5}$ or $b_{6}$ may suddenly change in magnitude.

\section{Results}

This section shows explicitly the role of resonant triads and weakly damped harmonic modes in the pattern selection problem for two-frequency forced Faraday waves. We examine how the cubic nonlinear coefficients in (16), for the Zhang-Viñals hydrodynamic equations vary as a function of $\theta_{h}$, the lattice angle and explain how this can be related to $\theta_{r}$, the resonant triad angle. The details of the computation of the coefficients are relegated to the Appendix. We focus on two examples, involving forcing frequency ratios $m / n=2 / 3$ and $6 / 7$. The $2 / 3$ case demonstrates the basic difference between the pattern selection problems for subharmonic and harmonic instabilities near the bicritical point. Our investigation also reveals a fundamental difference between harmonic wave pattern selection in the $2 / 3$ and $6 / 7$ cases, due to the presence of additional harmonic wave resonance tongues for the higher $6 / 7$ forcing frequencies; see Figure 1 .

\subsection{The Zhang-Viñals Hydrodynamic Equations}

The quadratic and cubic nonlinear coefficients in the hexagonal bifurcation problem (16) are computed in the appendix from a model of the two-frequency Faraday problem derived by Zhang and Viñals [3] from the Navier-Stokes equations. Their equations, which apply to weakly damped, small amplitude surface waves on a semi-infinite layer of fluid, describe the evolution of the surface 
height $h(\mathbf{x}, \tau)$ and surface velocity potential $\Phi(\mathbf{x}, \tau)$. Specifically,

$$
\begin{aligned}
\partial_{\tau} h= & \gamma \nabla^{2} h+\widehat{\mathcal{D}} \Phi-\nabla \cdot(h \nabla \Phi)+\frac{1}{2} \nabla^{2}\left(h^{2} \widehat{\mathcal{D}} \Phi\right)-\widehat{\mathcal{D}}(h \widehat{\mathcal{D}} \Phi)+\widehat{\mathcal{D}}\left[h \widehat{\mathcal{D}}(h \widehat{\mathcal{D}} \Phi)+\frac{1}{2} h^{2} \nabla^{2} \Phi\right] \\
\partial_{\tau} \Phi= & \gamma \nabla^{2} \Phi+\Gamma_{0} \nabla^{2} h-G(\tau) h+\frac{1}{2}(\widehat{D} \Phi)^{2}-\frac{1}{2}(\nabla \Phi)^{2}-(\widehat{\mathcal{D}} \Phi)\left[h \nabla^{2} \Phi+\widehat{\mathcal{D}}(h \widehat{\mathcal{D}} \Phi)\right] \\
& -\frac{1}{2} \Gamma_{0} \nabla \cdot\left((\nabla h)(\nabla h)^{2}\right),
\end{aligned}
$$

where $\widehat{\mathcal{D}}$ is a nonlocal operator that multiplies each Fourier component of a field by its wave number, i.e. $\widehat{\mathcal{D}} e^{i \mathbf{k} \cdot \mathbf{x}}=|\mathbf{k}| e^{i \mathbf{k} \cdot \mathbf{x}}$. Here time has been scaled by $\omega$ so that the (non-dimensionalized) two-frequency acceleration is

$$
G(\tau)=G_{0}-f(\cos (\chi) \cos (m \tau)+\sin (\chi) \cos (n \tau+\phi)) .
$$

The damping number $(\gamma)$, capillarity number $\left(\Gamma_{0}\right)$, gravity number $\left(G_{0}\right)$, and dimensionless acceleration $(f)$ are related to the forcing function (1) and the fluid parameters by

$$
\gamma \equiv \frac{2 \nu k_{0}^{2}}{\omega}, \quad \Gamma_{0} \equiv \frac{\Gamma k_{0}^{3}}{\rho \omega^{2}}, \quad G_{0} \equiv \frac{g_{0} k_{0}}{\omega^{2}}, \quad f \equiv \frac{g_{z} k_{0}}{\omega^{2}} .
$$

Here $\nu$ is the kinematic viscosity, $\Gamma$ is the surface tension, $\rho$ is the fluid density, and the wave number $k_{0}$ is chosen to satisfy the dispersion relation

$$
g_{0} k_{0}+\frac{\Gamma k_{0}^{3}}{\rho}=\left(\frac{m \omega}{2}\right)^{2} .
$$

\subsection{Example 1: $\mathrm{m} / \mathrm{n}=2 / 3$}

This example demonstrates a result of the general normal form analysis of Section 2.2, namely that proximity to the subharmonic/harmonic bicritical point will strongly influence the pattern selection problem for subharmonic waves, but not for harmonic waves. Specifically, we examine the cross-coupling coefficient $\beta(\theta)$ in (10) as a function of the angle $\theta$ for onset of both harmonic and subharmonic waves near the bicritical point. We show that only in the subharmonic case does $|\beta(\theta)|$ become large at the resonant angle $\theta_{r}$ in (3).

As described in Section 2.1, the primary instability changes from harmonic (Floquet multiplier +1 ) to subharmonic (Floquet multiplier -1 ) as $\chi$ in (21) is increased through the bicritical point $\chi_{c}$. This transition is determined from the linear hydrodynamic problem, which for the Zhang-Viñals model (20) takes the form of a damped Mathieu equation for each Fourier mode $h=h_{k}(\tau) e^{i k x}$ :

$$
h_{k}^{\prime \prime}+2 \gamma k^{2} h_{k}^{\prime}+\left(\gamma^{2} k^{4}+\Omega_{k}^{2}\right) h_{k}=f_{c} k[\cos (\chi) \cos (m \tau)+\sin (\chi) \cos (n \tau)] h_{k} .
$$

Here the natural frequency $\Omega_{k}$ satisfies the dispersion relation $\Omega_{k}^{2}=G_{0} k+\Gamma_{0} k^{3}$. A numericallycomputed neutral curve $f(k)$ for $m / n=2 / 3$ forcing and $\chi=\chi_{c}=66.6^{\circ}$ is given in Figure 4a. The other parameters of this example are $\phi=0^{\circ}, \Gamma_{0}=0.53, G_{0}=0.47$ and $\gamma=0.09$.

We now vary $\chi$ near $\chi_{c}$, holding all other parameters fixed, and examine the rhombic lattice cross-coupling coefficient $\beta(\theta)$ in (10) for onset subharmonic/harmonic waves, as appropriate. We 
have scaled the amplitudes $v_{1}$ and $v_{2}$ in (10) so that $a=-1$. We note that in the harmonic case $\beta$ diverges as $\theta \rightarrow 60^{\circ}$, i.e. when the rhombic lattice approaches the hexagonal one and there is an additional mode associated with the center manifold dynamics. This is in contrast to the subharmonic case, for which there is a normal form symmetry that ensures existence of a dynamically invariant subspace spanned by a pair of subharmonic modes separated by $60^{\circ}$. Thus in the subharmonic case $\beta$ remains finite at $\theta=60^{\circ}$.

For $\chi>\chi_{c}$ the primary instability is to subharmonic waves. For instance, for $\chi=66.7^{\circ}$ the minimum of the neutral curve occurs at wavenumber $k_{c, s}=1.415$ with forcing amplitude $f_{c}=0.842$, and is associated with a Floquet multiplier $\sigma=-1$. The nearly critical harmonic resonance tongue has its minimum at $(k, f)=(0.962,0.846)$. In this case, there is a spatio-temporally resonant triad comprised of the weakly damped harmonic mode and, from (3), two subharmonic modes separated by $\theta_{r}=39.9^{\circ}$. It follows from our general analysis of Section 2.2 that $\beta(\theta)$ will be large in magnitude for $\theta$ near $\theta_{r}$. Figure 5 a shows $\beta(\theta)$ for this case, and indeed, the nonlinear coefficient exhibits a large dip centered at $\theta=\theta_{r}=39.9^{\circ}$. At this angle, $|\beta(\theta)|$ takes on its largest value. Similar observations have been made by Zhang and Viñals 23 for forcing frequencies in ratio $m / n=1 / 2$.

In contrast, when $\chi<\chi_{c}$, so that the first instability to occur with increasing $f$ is harmonic, we find that the weakly damped subharmonic mode leaves no signature in the plot $\beta(\theta)$. For instance, for $\chi=66.5^{\circ}$ the primary instability is to harmonic waves at wavenumber $k_{c, h}=0.963$ and forcing amplitude $f_{c}=0.841$. The subhharmonic resonance tongue has a minimum at $(k, f)=$ $(1.415,0.843)$. While there is a spatially resonant triad involving two critical harmonic modes, which by (2) are separated by $\theta_{r}=85.7^{\circ}$, the triad of modes is not spatio-temporally resonant. Figure 5b shows the cross-coupling coefficient $\beta(\theta)$ for this case (with the region near $60^{\circ}$ removed). As anticipated, there is no signature of the weakly damped subharmonic mode in the plot. Similar observations have been made by Silber and Skeldon [26] in the setting of one-dimensional surface wave patterns.

\subsection{Example 2: $\mathrm{m} / \mathrm{n}=6 / 7$}

This example demonstrates a fundamental difference between harmonic wave pattern selection for low forcing frequencies (e.g., $2 \omega / 3 \omega)$ and for high forcing frequencies (e.g., $6 \omega / 7 \omega)$. This difference is due to the presence of multiple harmonic resonance tongues in the neutral curve associated with the higher forcing frequencies; see Figure 1 . In particular, these resonance tongues suggest the possibility that weakly damped harmonic modes may influence the harmonic wave pattern selection problem. This is in contrast to the $m / n=2 / 3$ example of the previous section, for which only subharmonic wave pattern competition was affected by weakly damped harmonic waves. In this section we also demonstrate that the weakly damped harmonic modes may stabilize harmonic wave superpatterns at a lattice angle $\theta_{h} \approx \theta_{r}$, due to a near cancellation of the two terms that contribute to $\beta\left(\theta_{r}\right)$ given by (9) as described in Section 2.2.

We focus on bifurcation to harmonic waves for $\chi=52.4^{\circ}$, which is close to the bicritical value $\chi_{c}=53.0^{\circ}$. The remaining parameters are $\phi=0^{\circ}, \Gamma_{0}=7.5, G_{0}=1.5$ and $\gamma=0.08$. We note that while the forcing frequency ratio $m / n=6 / 7$ coincides with that used in the experiments of Kudrolli, et al. [1], the remaining parameters do not coincide with the experiment. One problem with using the experimental parameters in the Zhang-Viñals equations is that the primary instability then moves to a subharmonic resonance tongue at very small wavenumber, i.e., the first resonance tongue of Figure $4 \mathrm{~b}$. This is because the Zhang-Viñals model does not accurately capture the 

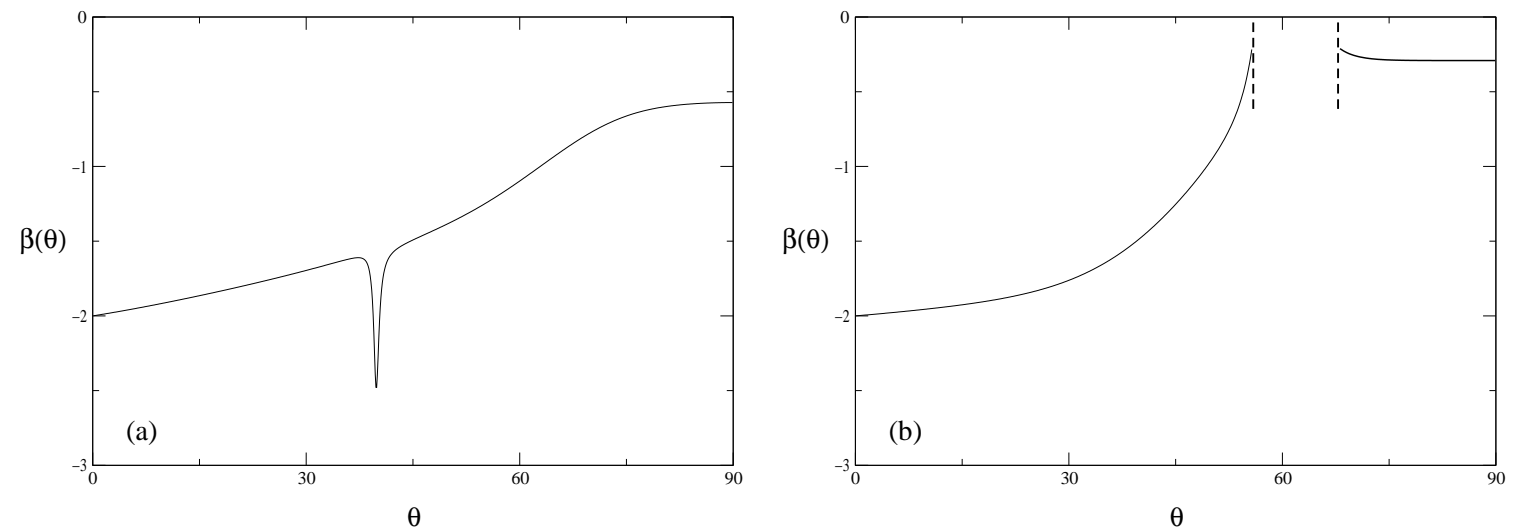

Figure 5: Cross coupling coefficients $\beta(\theta)$ in (10) computed in the Appendix from (20) for the case $m / n=2 / 3$ and $\phi=0^{\circ}$ in (21). The fluid parameters used are given in the caption of Figure Ga. (a) $\chi=66.7^{\circ}>\chi_{c}$, when the bifurcation is to subharmonic waves. Note the dip at $\theta=\theta_{r}=39.9^{\circ}$. (b) $\chi=66.5^{\circ}<\chi_{c}$, when the bifurcation is to harmonic waves. Because the (nearly) critical modes are not in temporal resonance, $\beta(\theta)$ shows no special structure at $\theta=\theta_{r}=85.7^{\circ}$. We have removed from this plot the region near $\theta=60^{\circ}$, where $\beta(\theta)$ diverges.

damping at small $k$ that is due to finite depth effects.

In this example we find two prominent features in the plot of the cross-coupling coefficient $\beta(\theta)$ in Figure 6a: a large dip at $\theta=67.6^{\circ}$ and a small spike at $\theta=22.2^{\circ}$. We now discuss the origin of these two features.

The large dip around $\theta=67.6^{\circ}$ is not a consequence of two-frequency forcing. Specifically, the dip remains in $\beta(\theta)$ even for purely $6 \omega$ forcing (i.e. in the limit $\chi \rightarrow 0) ; c f$. plots of $\beta(\theta)$ in Figures 6 a and $6 \mathrm{cc}$ which are obtained with $\chi=52.4^{\circ}$ and $\chi=0^{\circ}$, respectively. Thus this feature may be understood in the context of single frequency forcing, and has in fact already been investigated by Zhang and Viñals [3] in that setting. Specifically, if $\chi=0^{\circ}$ then the forcing period is $T^{\prime}=\frac{T}{6}=\frac{2 \pi}{6}$ and the primary instability is to subharmonic waves with period $2 T^{\prime}$. A plot of the corresponding neutral curve is given in Figure 6 $\mathrm{d}$, with the primary harmonic resonance tongue from Figure $4 \mathrm{~b}$ superimposed on it. In this single-frequency setting the feature at $67.6^{\circ}$ is understood as being due to the damped harmonic mode around $k=1.7$ in Figure Gd. Perhaps more relevant to this discussion is our observation that this feature, which leads to a large value of $\left|b_{4}+b_{5}+b_{6}\right|$, is destabilizing for superpatterns. To see this, we refer to the discussion surrounding equation (17) and to Figure 6b, which shows that

$$
\begin{aligned}
0>b_{1}+2 b_{2}>b_{4}+b_{5}+b_{6} & =\beta\left(\theta_{h}\right)+\beta\left(\theta_{h}+\frac{2 \pi}{3}\right)+\beta\left(\theta_{h}-\frac{2 \pi}{3}\right), \\
& =\beta\left(\theta_{h}\right)+\beta\left(\frac{\pi}{3}-\theta_{h}\right)+\beta\left(\frac{\pi}{3}+\theta_{h}\right) \text { for } \frac{\pi}{3}+\theta_{h} \approx 67.6^{\circ} .
\end{aligned}
$$

In contrast the spike at $\theta=22.2^{\circ}$ in Figure 6a minimizes $\left|b_{4}+b_{5}+b_{6}\right|$ at $\theta_{h} \approx 22.2^{\circ}$, as shown in Figure 6b. As we show below, this feature can lead to a stabilization of superpatterns and a destabilization of the simple hexagons. First we provide strong evidence that the spike is due to a 
resonance between the primary harmonic instability $(\sigma=1)$ and a weakly damped harmonic mode with a real Floquet multiplier $\mu$ that is close to 1 (see (5) and (9) of Section 2.2). In order to show this we must first compute the Floquet multipliers $\mu(k)$ at the critical forcing amplitude $f_{c}$ to determine the wavenumbers $k$ at which $\mu \approx 1$ at the onset of instability.

We determine the Floquet multipliers $\mu(k)$ at $f=f_{c}=1.552$ numerically from the linear problem (24). These are presented in Figure 7. We find that the multipliers are well approximated away from the two primary resonance tongues by considering the unforced problem $(f=0$ in equation 24), for which

$$
\mu_{ \pm}=e^{2 \pi \lambda_{ \pm}}, \quad \lambda_{ \pm}=-\gamma k^{2} \pm i \Omega_{k} .
$$

Figures $7 \mathrm{a}$ and $7 \mathrm{~b}$ show the magnitude $\xi$ and the phase $\psi$ of the Floquet multipliers $\mu=\xi e^{i \psi}$ both as computed numerically from (24) (solid line) and approximated by (26) (dotted line). Figure $7 \mathrm{c}$ shows the real part of the Floquet multipliers, $\xi \cos \psi$, versus wavenumber $k$. The "bubbles" in this plot correspond to wavenumbers at which the Floquet multipliers are real (as opposed to a complex conjugate pair). Weakly damped harmonic modes are associated with bubbles near a Floquet multiplier of +1 . Numerically we find that there are small bubbles of real Floquet multipliers whenever the phase $\psi$ is a multiple of $\pi$; this is demonstrated in Figure $7 \mathrm{~d}$. In particular, we find a bubble at wavenumber $k=0.383$, with associated real Floquet multiplier $\mu=0.93$. This mode is weakly damped and forms a resonant triad with primary harmonic modes separated by $\theta_{r}=22.2^{\circ}$. (Here $k_{c, h}=0.997$ for the primary instability, which corresponds to $k_{n}$ in (3), with $k_{m}=0.393$ determined by the weakly damped harmonic mode.) Here we have focused on the wavenumbers associated with real Floquet multipliers near $\mu=+1$ since weakly damped modes with complex Floquet multipliers do not form a spatio-temporally resonant triad with the primary harmonic modes.

We now present some hexagonal lattice bifurcation results for the specific parameters of this example, which are given in Figure $4 \mathrm{~b}$. The computation of the quadratic and cubic coefficients in the bifurcation problem (16) is described in the Appendix. We scale the amplitudes $z_{j}$ in (16) so that $b_{1}=-1$, in which case we find that $\epsilon=0.00014$ and $b_{2}=-2.73$. Thus we expect results of Section 2.3, which focused on the unfolding of the degenerate bifurcation problem $\epsilon=0$, to apply.

We find that simple hexagons, super hexagons and super triangles all bifurcate transcritically with the subcritical branch turning around in a saddle-node bifurcation. The stripes and rhombs solutions arise in supercritical pitchfork bifurcations. These claims are true for all lattice angles $\theta_{h}$ since the cubic coefficients $b_{1}, \ldots, b_{6}$ in $(16)$ are always negative; see Figure 6 a. Moreover, we find that simple hexagons are always stabilized in a saddle-node bifurcation and that they do not lose stability until after they reach the supercritical regime $\lambda>0$. In contrast, super hexagons and super triangles are always unstable at $\lambda=0$, since at that point the sign of the second eigenvalue in Table 1 is determined by $\operatorname{sgn}\left(b_{1}+2 b_{2}-3 b_{4}-3 b_{5}-3 b_{6}\right)$, which is positive for all $\theta$ (see Figure $6 \mathrm{~b}$ ). Thus, as $\lambda$ is increased through 0 , we expect a jump to finite amplitude simple hexagons as the other primary branches of (16) are unstable.

We find that simple hexagons eventually lose stability as $\lambda$ increases since the following two expressions from Table 1 change sign to positive (at least for some $\theta_{h}$ )

$$
\operatorname{sgn}\left(-\epsilon x+\left(b_{1}-b_{2}\right) x^{2}\right), \quad \operatorname{sgn}\left(-\epsilon x+\left(b_{4}+b_{5}+b_{6}-b_{1}-2 b_{2}\right) x^{2}\right) .
$$

as the amplitude $x$ of simple hexagons grows with $\lambda$. The first quantity changes from negative to positive at $\lambda \approx 3.2 \times 10^{-8}$. The second quantity changes sign with increasing $\lambda$ only for those values 

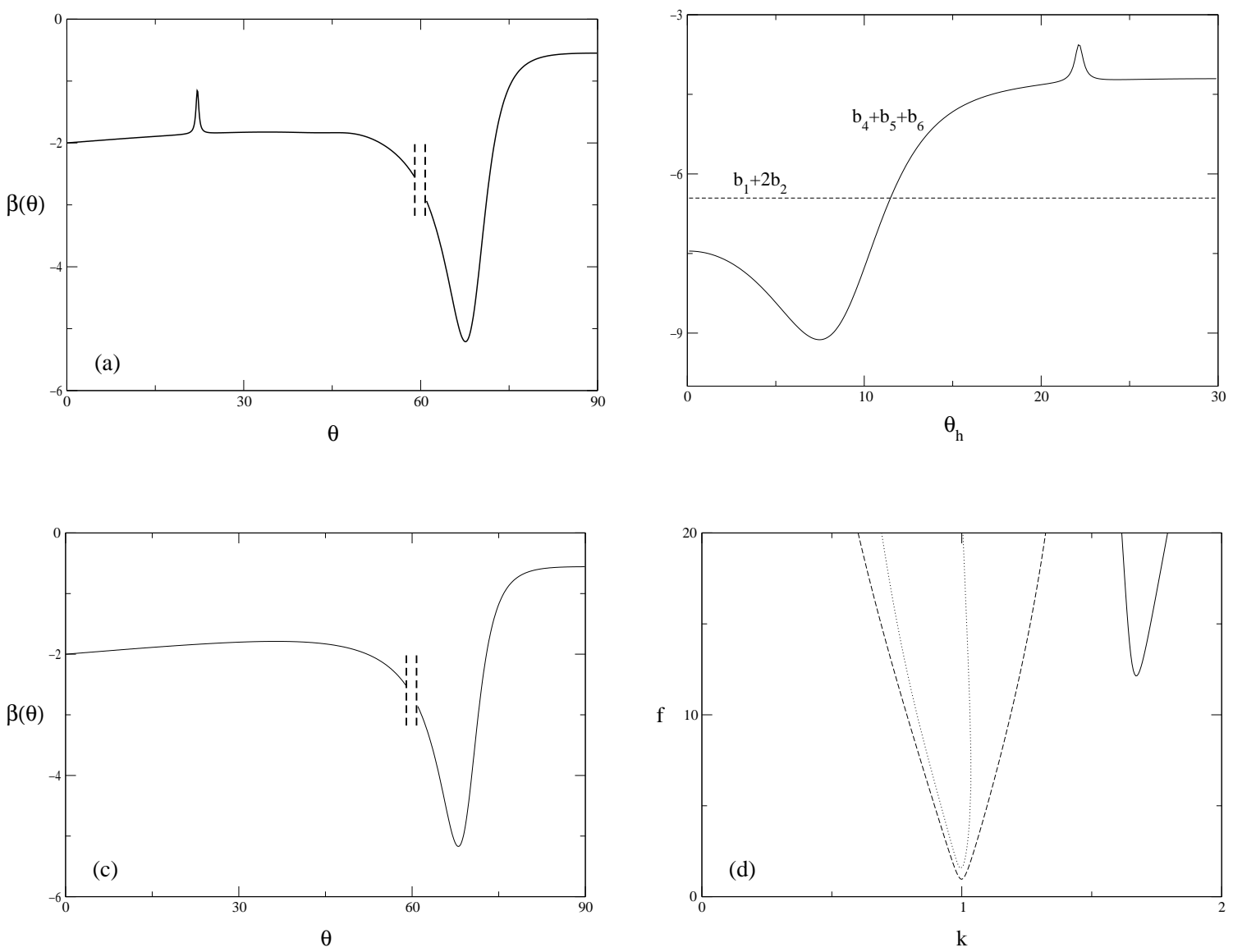

Figure 6: (a) Cross-coupling coefficient $\beta(\theta)$ in (10) computed from (20) for the case $m / n=6 / 7$, $\phi=0^{\circ}$ and $\chi=52.4^{\circ}<\chi_{c}$ in (21), and for fluid parameters given in the caption of Figure $4 \mathrm{~b}$. (b) Plots of $b_{1}+2 b_{2}$ (dashed) and $b_{4}+b_{5}+b_{6}$ (solid) versus $\theta_{h}$. We note that $\theta_{h}$ only takes on the discrete values satisfying (14). (c) Cross-coupling coefficient $\beta(\theta)$ for $6 \omega$ forcing only; we have used the same parameters as in (a) except that now $\chi=0^{\circ}$. (d) Neutral curve for single frequency forcing. Floquet multipliers of $+1(-1)$ are indicated by solid (dashed) lines, and are computed relative to the period $T^{\prime}=2 \pi / 6$. The primary harmonic resonance tongue from the two-frequency case of Figure $4 \mathrm{~b}$ is superimposed as a dotted line. 

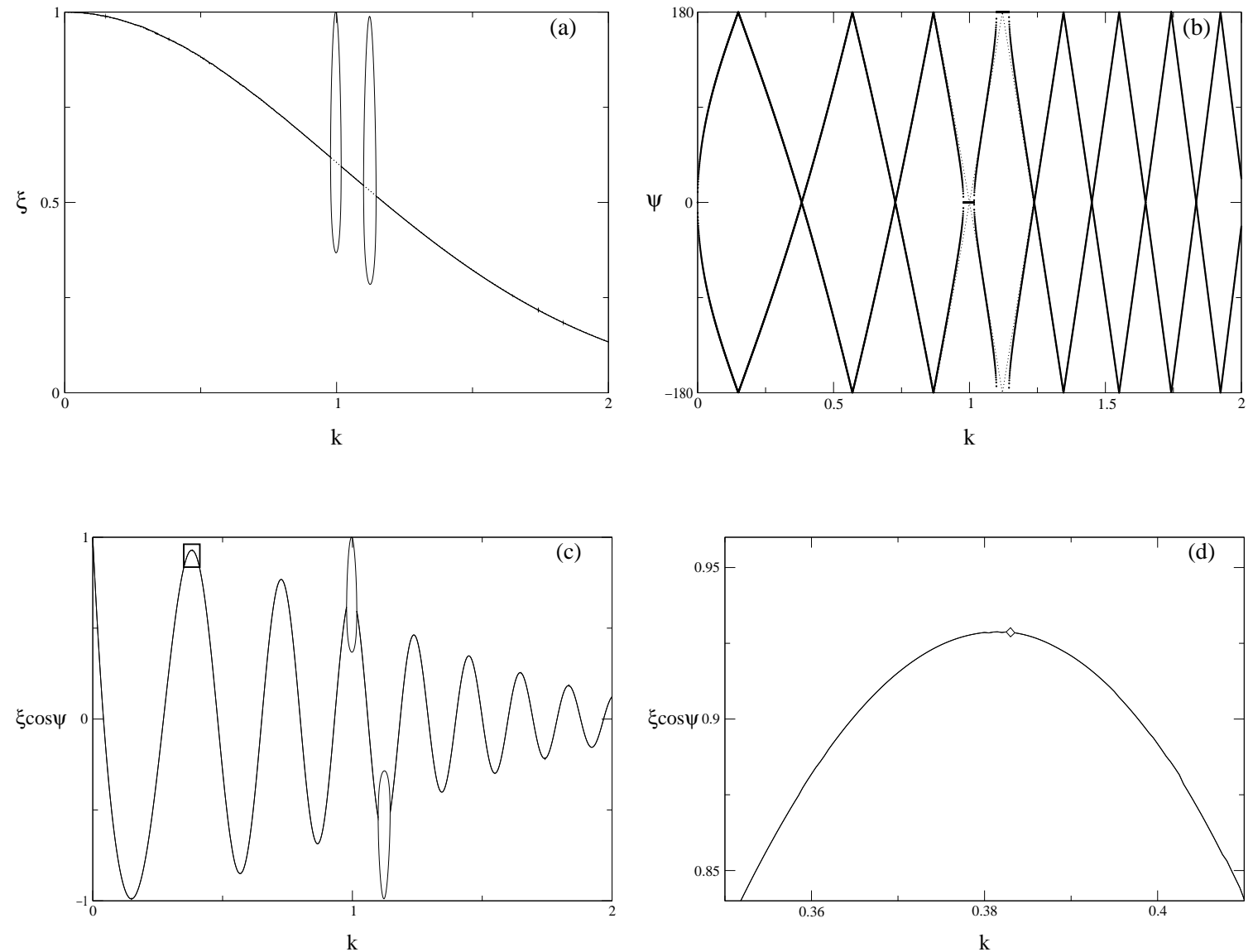

Figure 7: Floquet multipliers $\mu=\xi e^{i \psi}$ computed from (24) for the parameters used in Figure $4 \mathrm{~b}$ and for critical forcing amplitude $f=f_{c}=1.552$. (a) Magnitude $\xi$, and (b) phase $\psi v s$. wavenumber $k$. The solid lines in (a) and (b) are computed numerically, while the the dotted lines are obtained by considering the unforced problem $f=0$; see equation 26. (c) Numerically computed real part $\xi \cos \psi$ of the Floquet multipliers. The "bubbles" correspond to real-valued Floquet multipliers. The boxed region, shown blown up in (d), reveals a tiny "bubble" around $k=0.383$, with real Floquet multiplier $\mu=0.93$. 


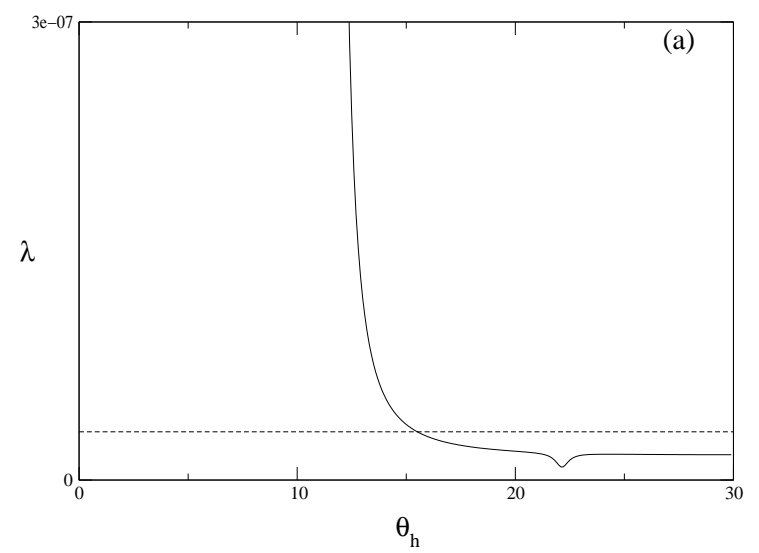

(b)

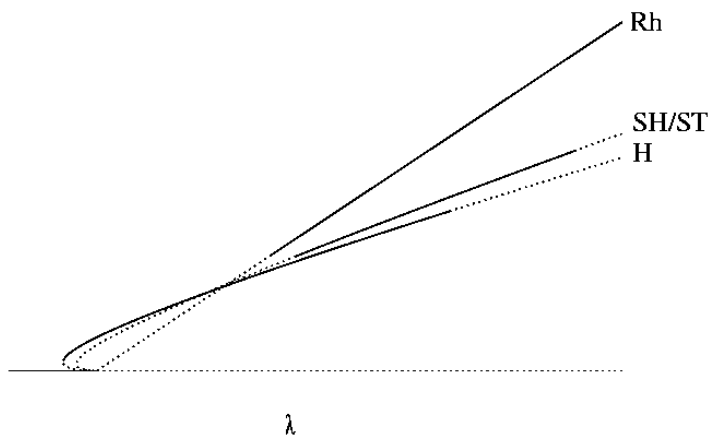

Figure 8: (a) $\lambda$ value at which the first (dashed) and second (solid) eigenvalue expressions in (27) turn positive versus the lattice angle $\theta_{h}$. Note that simple hexagons $(\mathrm{H})$ first lose stability to perturbations in the super hexagon/triangle $(\mathrm{SH} / \mathrm{ST})$ direction at $\theta_{h} \approx 22.2^{\circ}$. (b) Schematic bifurcation diagram for the $\left(n_{1}, n_{2}\right)=(3,2)$ lattice of Figure 3, which corresponds to $\theta_{h}=21.8^{\circ}$. Stable (unstable) solutions are indicated by a solid (dotted) line. We do not show secondary branches or primary branches that are never stable. The stable rhombs solution (Rh) corresponds to one with an angle of $81.8^{\circ}$, which is the rhombs solution closest to $90^{\circ}$ for this hexagonal lattice. The other two rhombs solutions are unstable.

of $\theta_{h}$ where $b_{4}+b_{5}+b_{6}-b_{1}-2 b_{2}>0$, a condition which is met for $\theta_{h} \geq 11.5^{\circ}$. Figure 8a shows the value of $\lambda$ where the expressions of (27) change sign as a function of $\theta_{h}$. It follows that simple hexagons lose stability first on the lattice with angle $\theta_{h} \approx 22.2^{\circ}$. This instability has an associated eigenvector in the direction of super hexagon/triangles, and at this value of $\lambda$, super hexagons (or triangles) are stable. These results are summarized in Figure $8 \mathrm{~b}$, which shows part of the bifurcation diagram computed for the hexagonal lattice with $\left(n_{1}, n_{2}\right)=(3,2)$, which corresponds to an angle $\theta_{h}=21.8^{\circ}$. Note that when simple hexagons lose stability, both rhombs (Rh) and a superpattern are stable. Because the instability that first destabilizes the simple hexagons is in the direction of a superpattern with $\theta_{h} \approx 22.2^{\circ}$, we expect that the transition would be a hysteretic one involving the simple hexagons and a superpattern, at least in the absence of noise and other imperfections. We cannot determine whether the superpattern is hexagonal or triangular from our calculations, since this requires knowledge of fifth order terms in the bifurcation problem 34.

\section{Conclusions}

In this paper we have examined the effect of spatio-temporally resonant triads on two-dimensional pattern selection in parametrically excited systems. Using a normal form transformation to enforce temporal symmetry and center manifold reduction, we have argued that weakly damped harmonic modes can strongly influence pattern selection by causing certain cubic cross-coupling coefficients in a twelve-dimensional $\mathrm{D}_{6}+\mathrm{T}^{2}$-equivariant bifurcation problem to suddenly vary in magnitude for certain lattice angles $\theta_{h}$. This suggests an important consideration in choosing one over another of 
the countable set of twelve-dimensional representations relevant to hexagonal bifurcation problems. Weakly damped subharmonic modes, on the other hand, do not have such an effect.

Our general analysis applies to any parametrically excited pattern forming system, but in particular is relevant to the interpretation of many recent experiments on two-frequency forced Faraday waves. In such experiments, a bicritical point exists where subharmonic and harmonic instabilities are simultaneously excited. On one side of the bicritical point, a subharmonic mode is excited and there is a weakly damped harmonic mode, while on the other, it is the harmonic mode which is excited and the subharmonic mode which is weakly damped. We showed that this weakly damped subharmonic mode does not influence the harmonic wave pattern selection problem.

We have derived the quadratic and cubic coefficients in the rhombic and hexagonal bifurcation equations describing the onset of patterns from the hydrodynamic equations of Zhang and Viñals. We presented results for two different sets of parameters. In the first case, the two forcing frequencies are in the ratio $2 / 3$ and the modes near the bicritical point are the only ones of relevance. As expected from our normal form analysis, for subharmonic waves, the weakly damped harmonic mode affects the cross-coupling coefficients, while for harmonic waves, the weakly damped subharmonic mode had no effect.

In the second case of $6 / 7$ forcing we have shown that, in addition to the modes near the bicritical point, there are other harmonic modes that are important. These modes are not close to onset in the sense that they only become critical at a much higher value of the excitation amplitude, but are weakly damped and thus must be taken into account. We demonstrate that they can have a stabilizing effect on superlattice patterns at a lattice angle approximately equal to the angle of the harmonic-harmonic resonance. This can occur if the contribution of these weakly damped modes to the nonlinear cross-coupling coefficient nearly cancels the other contributions to this term, and hence is a subtle effect that depends on certain details of the nonlinear problem, as well as the results of the linear analysis which identifies the near critical modes. For the parameters we have chosen, the onset pattern is simple hexagons, but upon a further increase of the forcing, there is an instability to a superlattice pattern associated with a hexagonal lattice with $\left(n_{1}, n_{2}\right)=(3,2)$.

The experiments of Kudrolli, Pier and Gollub [1] found a superlattice pattern near the bicritical point which sits on a lattice with $\left(n_{1}, n_{2}\right)=(3,2)$. The work in this paper suggests that the observation of this pattern could be explained by the interaction of the primary harmonic instability and weakly damped harmonic modes. However, the Zhang-Viñals equations are not valid in the parameter regime where this experiment was performed, and thus a study of the full hydrodynamic problem is necessary to confirm this conjecture. A complete study should also involve a more complete analysis of the codimension-2 bifurcation point and the associated dynamics, in the spirit of J.D. Crawford's early work on competing instabilities in the Faraday problem [27]. This would be of interest in light of recent two-frequency experiments by Arbell and Fineberg [11] that show a variety of dynamic states near the bicritical point, which involve both critical modes.

\section{A Perturbation Theory}

Here we outline the computation of the coefficients in (10) and (16) from the equations of Zhang and Viñals (20). A multiple-scale perturbation method is used to derive expressions for the coefficients which are then evaluated numerically using a pseudospectral approach. This follows closely the method described in [26] for the onset of one-dimensional patterns and we refer the reader there 
for further details.

The coefficients can be derived by considering two different calculations, namely the bifurcation problem (16) restricted in turn to a rhombic and a simple hexagons subspace.

\section{A.1 Rhombic lattice computation}

In order to compute the coefficient $a$ and the cross-coupling coefficient $\beta(\theta)$ in $(10)$ we seek solutions which are periodic on a rhombic lattice associated with an angle $\theta$. We are thereby able to compute the coefficients $b_{1}, b_{4}, b_{5}$, and $b_{6}$ in the bifurcation equations (16) since $b_{1}=a, b_{4}=\beta\left(\theta_{h}\right)$, $b_{5}=\beta\left(\theta_{h}+2 \pi / 3\right)$, and $b_{6}=\beta\left(\theta_{h}-2 \pi / 3\right)$.

First we introduce a small parameter $\eta$, such that

$$
\begin{aligned}
h(x, y, \tau)= & \eta h_{1}(x, y, \tau, T)+\eta^{2} h_{2}(x, y, \tau, T) \\
& +\eta^{3} h_{3}(x, y, \tau, T)+\cdots \\
\Phi(x, y, \tau)= & \eta \Phi_{1}(x, y, \tau, T)+\eta^{2} \Phi_{2}(x, y, \tau, T) \\
& +\eta^{3} \Phi_{3}(x, y, \tau, T)+\cdots
\end{aligned}
$$

in (20) where

$$
T=\eta^{2} \tau, \quad f=f_{c}+\eta^{2} f_{2} .
$$

Here $f_{c}$ is the critical excitation amplitude. The terms in the expansion for $h$ and $\Phi$ may be written in the following separable Floquet-Fourier form:

$$
\begin{aligned}
h_{1}= & {\left[w_{1}(T) e^{i k_{c} x}+w_{4}(T) e^{i k_{c}(c x+s y)}+c . c .\right] p_{1}(\tau) } \\
\Phi_{1}= & {\left[w_{1}(T) e^{i k_{c} x}+w_{4}(T) e^{i k_{c}(c x+s y)}+c . c .\right] q_{1}(\tau) } \\
h_{2}= & {\left[w_{1}^{2}(T) e^{2 i k_{c} x}+w_{4}^{2}(T) e^{2 i k_{c}(c x+s y)}\right] p_{2,1}(\tau) } \\
& +w_{1}(T) \bar{w}_{4}(T) e^{i k_{c}((1-c) x-s y)} p_{2,2}(\tau) \\
& +w_{1}(T) w_{4}(T) e^{i k_{c}((1+c) x+s y)} p_{2,3}(\tau)+c . c . \\
\Phi_{2}= & {\left[w_{1}^{2}(T) e^{2 i k_{c} x}+w_{4}^{2}(T) e^{2 i k_{c}(c x+s y)}\right] q_{2,1}(\tau) } \\
& +w_{1}(T) \bar{w}_{4}(T) e^{i k_{c}((1-c) x-s y)} q_{2,2}(\tau) \\
& +w_{1}(T) w_{4}(T) e^{i k_{c}((1+c) x+s y)} q_{2,3}(\tau)+\text { c.c. }
\end{aligned}
$$

where $c=\cos \theta, s=\sin \theta$, and $\theta$ is not a multiple of $\frac{\pi}{3}$. Here $p_{1}$ and $q_{1}$ are real $2 \pi$-periodic functions of the fast time $\tau$ in the case of harmonic waves; in the case of subharmonic waves they are $4 \pi$-periodic in $\tau$. Additionally, $p_{2, r}$ and $q_{2, r}(r=1,2,3)$ are real $2 \pi$-periodic functions of $\tau$. The wave number $k_{c}$ is associated with the onset unstable mode.

At $\mathcal{O}(\eta)$ we recover the linear problem which determines $k_{c}$ and $f_{c}$, as well as the functions $p_{1}$, $q_{1}$ to within a multiplicative constant. At $\mathcal{O}\left(\eta^{2}\right)$, equations are found which allow us to solve for the functions $p_{2, r}$ and $q_{2, r}$. Finally, at $\mathcal{O}\left(\eta^{3}\right)$, we apply a solvability condition to ensure that a periodic solution exists. This condition leads to the amplitude equations

$$
\begin{aligned}
& \delta \frac{d w_{1}}{d T}=\alpha f_{2} w_{1}+A\left|w_{1}\right|^{2} w_{1}+B(\theta)\left|w_{4}\right|^{2} w_{1} \\
& \delta \frac{d w_{4}}{d T}=\alpha f_{2} w_{4}+A\left|w_{4}\right|^{2} w_{4}+B(\theta)\left|w_{1}\right|^{2} w_{4},
\end{aligned}
$$


where

$$
\begin{aligned}
& \delta= \frac{1}{2 \pi} \int_{0}^{4 \pi}\left(p_{1}^{\prime}+\gamma k_{c}^{2} p_{1}\right) \widetilde{p}_{1} d \tau \\
& \alpha=\frac{k_{c}}{4 \pi} \int_{0}^{4 \pi}[\cos (\chi) \cos (m \tau)+\sin (\chi) \cos (n \tau+\phi)] p_{1} \widetilde{p}_{1} d \tau \\
& A=\frac{k_{c}^{2}}{4 \pi} \int_{0}^{4 \pi}\left[-k_{c}\left(p_{1}^{2} q_{1}\right)^{\prime}-\gamma k_{c}^{3} p_{1}^{2} q_{1}-2\left(q_{1} p_{2,1}\right)^{\prime}-2 \gamma k_{c}^{2} q_{1} p_{2,1}\right. \\
&\left.\quad+k_{c}^{2} q_{1}^{2} p_{1}+\frac{3}{2} k_{c}^{3} \Gamma_{0} p_{1}^{3}\right] \widetilde{p}_{1} d \tau \\
& B(\theta)=\frac{k_{c}^{2}}{4 \pi} \int_{0}^{4 \pi}\left[(1-c-\sqrt{2-2 c})\left[\left(p_{1} q_{2,2}\right)^{\prime}+\gamma k_{c}^{2} p_{1} q_{2,2}-k_{c} q_{1} q_{2,2}\right]\right. \\
& \quad+(1+c-\sqrt{2+2 c})\left[\left(p_{1} q_{2,3}\right)^{\prime}+\gamma k_{c}^{2} p_{1} q_{2,3}-k_{c} q_{1} q_{2,3}\right] \\
& \quad-(1-c)\left[\left(p_{2,2} q_{1}\right)^{\prime}+\gamma k_{c}^{2} p_{2,2} q_{1}\right]-(1+c)\left[\left(p_{2,3} q_{1}\right)^{\prime}+\gamma k_{c}^{2} p_{2,3} q_{1}\right] \\
& \quad-(6-2 \sqrt{2-2 c}-2 \sqrt{2+2 c})\left[k_{c}\left(p_{1}^{2} q_{1}\right)^{\prime}+\gamma k_{c}^{3} p_{1}^{2} q_{1}-k_{c}^{2} p_{1} q_{1}^{2}\right] \\
&\left.\left.\quad+\Gamma_{0}\left(3 c^{2}+s^{2}\right) k_{c}^{3} p_{1}^{3}\right)\right] \widetilde{p}_{1} d \tau .
\end{aligned}
$$

In the above, a prime denotes differentiation with respect to $\tau$ and $\widetilde{p}_{1}$ is the equivalent of $p_{1}$ for the adjoint problem at $\mathcal{O}(\eta)$. The amplitude equations (31) may be re-scaled and then comparison with the map (10) yields

$$
a=b_{1}=\operatorname{sgn}(A \alpha), \quad \beta(\theta)=\operatorname{sgn}(A \alpha) \frac{B(\theta)}{A} .
$$

\section{A.2 Hexagonal lattice computation}

Similarly, we compute the coefficients $\epsilon$ and $b_{2}$ in the bifurcation equations (16) by seeking solutions in the form of simple hexagons. Here we use a three-timing perturbation method, writing the solution as

$$
\begin{aligned}
h(x, y, \tau)= & \eta h_{1}\left(x, y, \tau, T_{1}, T_{2}\right)+\eta^{2} h_{2}\left(x, y, \tau, T_{1}, T_{2}\right) \\
& +\eta^{3} h_{3}\left(x, y, \tau, T_{1}, T_{2}\right)+\cdots \\
\Phi(x, y, \tau)= & \eta \Phi_{1}\left(x, y, \tau, T_{1}, T_{2}\right)+\eta^{2} \Phi_{2}\left(x, y, \tau, T_{1}, T_{2}\right) \\
& +\eta^{3} \Phi_{3}\left(x, y, \tau, T_{1}, T_{2}\right)+\cdots,
\end{aligned}
$$

where

$$
T_{1}=\eta \tau, \quad T_{2}=\eta^{2} \tau,
$$

and

$$
\begin{aligned}
& h_{1}=w_{1}\left(T_{1}, T_{2}\right) p_{1}(\tau)\left[e^{i k_{c} x}+e^{i k_{c}\left(-\frac{1}{2} x+\frac{\sqrt{3}}{2} y\right)}+e^{i k_{c}\left(-\frac{1}{2} x-\frac{\sqrt{3}}{2} y\right)}+c . c .\right] \\
& \Phi_{1}=w_{1}\left(T_{1}, T_{2}\right) q_{1}(\tau)\left[e^{i k_{c} x}+e^{i k_{c}\left(-\frac{1}{2} x+\frac{\sqrt{3}}{2} y\right)}+e^{i k_{c}\left(-\frac{1}{2} x-\frac{\sqrt{3}}{2} y\right)}+c . c .\right] \\
& h_{2}=w_{1}^{2}\left(T_{1}, T_{2}\right)\left\{p_{2,1}(\tau)\left[e^{i k_{c} 2 x}+e^{i k_{c}(-x+\sqrt{3} y)}+e^{i k_{c}(-x-\sqrt{3} y)}+c . c .\right]\right.
\end{aligned}
$$




$$
\begin{aligned}
& +p_{2,2}(\tau)\left[e^{i k_{c} x}+e^{i k_{c}\left(-\frac{1}{2} x+\frac{\sqrt{3}}{2} y\right)}+e^{i k_{c}\left(-\frac{1}{2} x-\frac{\sqrt{3}}{2} y\right)}+c . c .\right] \\
& \left.+p_{2,3}(\tau)\left[e^{i k_{c}\left(\frac{3}{2} x-\frac{\sqrt{3}}{2} y\right)}+e^{i k_{c} \sqrt{3} y}+e^{i k_{c}\left(\frac{3}{2} x+\frac{\sqrt{3}}{2} y\right)}+c . c .\right]\right\} \\
\Phi_{2}= & w_{1}^{2}\left(T_{1}, T_{2}\right)\left\{q_{2,1}(\tau)\left[e^{i k_{c} 2 x}+e^{i k_{c}(-x+\sqrt{3} y)}+e^{i k_{c}(-x-\sqrt{3} y)}+\text { c.c. }\right]\right. \\
& +q_{2,2}(\tau)\left[e^{i k_{c} x}+e^{i k_{c}\left(-\frac{1}{2} x+\frac{\sqrt{3}}{2} y\right)}+e^{i k_{c}\left(-\frac{1}{2} x-\frac{\sqrt{3}}{2} y\right)}+c . c .\right] \\
& \left.+q_{2,3}(\tau)\left[e^{i k_{c}\left(\frac{3}{2} x-\frac{\sqrt{3}}{2} y\right)}+e^{i k_{c} \sqrt{3} y}+e^{i k_{c}\left(\frac{3}{2} x+\frac{\sqrt{3}}{2} y\right)}+c . c .\right]\right\} .
\end{aligned}
$$

As with the rhombic case, $p_{1}, q_{1}, p_{2, r}$ and $q_{2, r}$ are real. Additionally, we take the amplitude $w_{1}\left(T_{1}, T_{2}\right)$ to be real.

For the harmonic case, at $\mathcal{O}\left(\eta^{2}\right)$ the solvability condition,

$$
\delta \frac{\partial w_{1}}{\partial T_{1}}=\beta_{0} w_{1}^{2},
$$

must be satisfied, where $\delta$ is given by (32). The quadratic coefficient is

$$
\beta_{0}=\frac{k_{c}^{2}}{4 \pi} \int_{0}^{4 \pi}\left[-\left(p_{1} q_{1}\right)^{\prime}-\gamma k_{c}^{2} p_{1} q_{1}+\frac{1}{2} k_{c} q_{1}^{2}\right] \widetilde{p_{1}} d \tau .
$$

There is no solvability condition for subharmonic waves at $\mathcal{O}\left(\eta^{2}\right)$, reflecting the fact that there are no even terms in the amplitude equations (16) for this case.

At $\mathcal{O}\left(\eta^{3}\right)$, we again apply a solvability condition to ensure that a periodic solution exists. This conditions leads to the amplitude equation

$$
\delta \frac{\partial w_{1}}{\partial T_{2}}=\alpha f_{2} w_{1}+\left(A+2 \beta_{2}\right) w_{1}^{3}
$$

The coefficients $\delta, \alpha$, and $A$ are given by (32), and

$$
\begin{aligned}
\beta_{2}=\frac{1}{4 \pi} \int_{0}^{4 \pi} & {\left[\left(\frac{3}{2}-\sqrt{3}\right) k_{c}^{2}\left[\left(p_{1} q_{2,3}\right)^{\prime}+\gamma k_{c}^{2} p_{1} q_{2,3}-k_{c} q_{1} q_{2,3}\right]\right.} \\
& +(2 \sqrt{3}-4) k_{c}^{3}\left[\left(p_{1}^{2} q_{1}\right)^{\prime}+\gamma k_{c}^{2} p_{1}^{2} q_{1}-k_{c} p_{1} q_{1}^{2}\right] \\
& -\frac{3}{2} k_{c}^{2}\left[\left(p_{2,3} q_{1}\right)^{\prime}+\gamma k_{c}^{2} p_{2,3} q_{1}-\Gamma_{0} k_{c}^{3} p_{1}^{3}\right] \\
& -\frac{1}{2} k_{c}^{2}\left[\left(p_{1} q_{2,2}\right)^{\prime}+\gamma k_{c}^{2} p_{1} q_{2,2}+\left(p_{2,2} q_{1}\right)^{\prime}+\gamma k_{c}^{2} p_{2,2} q_{1}-k_{c} q_{1} q_{2,2}\right] \\
& \left.-\frac{\beta_{0}}{\delta}\left[k_{c}^{2} p_{1} q_{1}+\frac{\beta_{0}}{\delta} p_{1}+2 p_{2,2}^{\prime}+2 \gamma k_{c}^{2} p_{2,2}\right]\right] \widetilde{p}_{1} d \tau .
\end{aligned}
$$

By rescaling $\eta w_{j}\left(T_{1}, T_{2}\right) \rightarrow w_{j}(T)$ and $\eta^{2} \alpha f_{2} \rightarrow \alpha f_{2}$, we obtain the reconstituted hexagonal bifurcation equation

$$
\delta \frac{d w_{1}}{d T}=\alpha f_{2} q_{1}+\beta_{0} w_{1}^{2}+\left(A+2 \beta_{2}\right) w_{1}^{3}
$$

Finally, after rescaling as for the rhombic case, and comparing (41) to (16) we find that

$$
\epsilon=\operatorname{sgn}(\alpha) \frac{\beta_{0}}{\sqrt{|\alpha A|}}, \quad b_{2}=\operatorname{sgn}(A \alpha) \frac{\beta_{2}}{A} .
$$




\section{Acknowledgments}

First and foremost, we acknowledge the tremendous influence of John David Crawford's research on parametrically excited wave patterns on our own study of Faraday waves. MS also wishes to acknowledge the inspiration she has always taken from John David's work, as well as the encouragement she received from him.

The research of MS was supported by NSF grant DMS-9972059 and by an NSF CAREER award DMS-9502266.

\section{References}

[1] A. Kudrolli, B. Pier, and J.P. Gollub. Superlattice patterns in surface waves. Physica D, 123:99-111, 1998.

[2] M. Silber and M.R.E. Proctor. Nonlinear competition between small and large hexagonal patterns. Phys. Rev. Lett., 81:2450-2453, 1998.

[3] W. Zhang and J. Viñals. Pattern formation in weakly damped parametric surface waves. J. Fluid Mech., 336:301-330, 1997.

[4] J. Bechhoefer, V. Ego, S. Manneville, and B. Johnson. An experimental study of the onset of parametrically pumped surface waves in viscous fluids. J. Fluid Mech., 288:325-350, 1995.

[5] A. Kudrolli and J.P. Gollub. Patterns and spatiotemporal chaos in parametrically forced surface waves: A systematic survey at large aspect ratio. Physica D, 97:133-154, 1996.

[6] H.W. Müller. Periodic triangular patterns in the Faraday experiment. Phys. Rev. Lett., 71:3287-3290, 1993.

[7] W.S. Edwards and S. Fauve. Patterns and quasi-patterns in the Faraday experiment. J. Fluid Mech., 278:123-148, 1994.

[8] B. Christiansen, P. Alstrom, and M.T. Levinsen. Ordered capillary-wave states: Quasicrystals, hexagons, and radial waves. Phys. Rev. Lett., 68:2157-2160, 1992.

[9] C. Wagner, H.W. Müller, and K. Knorr. Faraday waves on a viscoelastic liquid. Phys. Rev. Lett., 83:308-311, 1999.

[10] H. Arbell and J. Fineberg. Spatial and temporal dynamics of two interacting modes in parametrically driven surface waves. Phys. Rev. Lett., 81:4384-4387, 1998.

[11] H. Arbell and J. Fineberg. Two-mode rhomboidal states in driven surface waves. Phys. Rev. Lett., 84:654-657, 2000.

[12] H. Arbell and J. Fineberg. Temporally harmonic oscillons in Newtonian fluids. preprint, 2000.

[13] H.W. Müller, R. Friedrich, and D. Papathanassiou. Theoretical and experimental investigations of the Faraday instability. In F. Busse and S.C. Müller, editors, Evolution of Spontaneous Structures in Dissipative Continuous Systems, Lecture Notes in Physics, pages 231-265. Springer, 1998. 
[14] M. Faraday. On the forms and states of fluids on vibrating elastic surfaces. Phil. Trans. R. Soc. Lond., 121:319-340, 1831.

[15] T.B. Benjamin and F. Ursell. The stability of a plane free surface of a liquid in vertical periodic motion. Proc. R. Soc. Lond. A, 225:505-515, 1954.

[16] K. Kumar. Linear theory of Faraday instability in viscous liquids. Proc. Roy. Soc. Lond. A, 452:1113-1126, 1996.

[17] H.W. Müller, H. Wittmer, C.Wagner, J. Albers, and K. Knorr. Analytic stability theory for Faraday waves and the obervation of the harmonic surface response. Phys. Rev. Lett., 78:2357-2360, 1997.

[18] E. Cerda and E. Tirapegui. Faraday's instability for viscous fluids. Phys. Rev. Lett., 78:859$862,1997$.

[19] J. Beyer and R. Friedrich. Faraday instability: Linear analysis for viscous fluids. Phys. Rev. E, 51:1162-1168, 1995.

[20] T. Besson, W.S. Edwards, and L. Tuckerman. Two-frequency parametric excitation of surface waves. Phys. Rev. E, 54:507-513, 1996.

[21] D. Binks and W. van de Water. Nonlinear pattern formation of Faraday waves. Phys. Rev. Lett., 78:4043-4046, 1997.

[22] D. Binks, M.-T. Westra, and W. van de Water. Effect of depth on the pattern formation of Faraday waves. Phys. Rev. Lett., 79:5010-5013, 1997.

[23] W. Zhang and J. Viñals. Pattern formation in weakly damped parametric surface waves driven by two frequency components. J. Fluid Mech., 341:225-244, 1997.

[24] R. Lifshitz and D.M. Petrich. Theoretical model for Faraday waves with multiple-frequency forcing. Phys. Rev. Lett., 79:1261-1264, 1997.

[25] P. Chen and J. Viñals. Amplitude equations and pattern selection in Faraday waves. Phys. Rev. E, 60:559-570, 1999.

[26] M. Silber and A.C. Skeldon. Parametrically excited surface waves: Two-frequency forcing, normal form symmetries, and pattern selection. Phys. Rev. E, 59:5446-5456, 1999.

[27] J.D. Crawford, E. Knobloch, and H. Riecke. Period-doubling mode interactions with circular symmetry. Physica D, 44:340-396, 1990.

[28] J.D. Crawford. Normal forms for driven surface waves: Boundary conditions, symmetry, and genericity. Physica D, 52:429-457, 1991.

[29] J.D. Crawford. Surface waves in nonsquare containers with square symmetry. Phys. Rev. Lett., 67:441-444, 1991.

[30] J.D. Crawford, J.P. Gollub, and D. Lane. Hidden symmetries of parametrically forced waves. Nonlinearity, 6:119-164, 1993. 
[31] M. Golubitsky, I. Stewart, and D.G. Schaeffer. Singularities and Groups in Bifurcation Theory: Vol. II. Number 69 in Appl. Math. Sci. Ser. Springer-Verlag, New York, 1988.

[32] J.D. Crawford and E. Knobloch. Symmetry and symmetry-breaking bifurcations in fluid dynamics. Ann. Rev. Fluid Mech., 23:341-387, 1991.

[33] B. Dionne and M. Golubitsky. Planforms in two and three dimensions. Z. Angew. Math. Phys., 43:36-62, 1992.

[34] B. Dionne, M. Silber, and A.C. Skeldon. Stability results for steady, spatially-periodic planforms. Nonlinearity, 10:321-353, 1997.

[35] J.D. Crawford. Introduction to bifurcation theory. Rev. Mod. Phys., 63:991-1037, 1991. 